\title{
Development and Characterization of Patient-Derived Xenografts from Non-Small Cell Lung Cancer Brain Metastases
}

Andrew M. Baschnagel, M.D., ${ }^{1,2}$ Saakshi Kaushik, M.S., ${ }^{1}$ Arda Durmaz, M.S., ${ }^{3}$ Steve Goldstein, Ph.D., ${ }^{2,4}$ Irene M. Ong, Ph.D., ${ }^{2,4,5}$ Lindsey Abel, B.S., ${ }^{1}$ Paul A. Clark, Ph.D., ${ }^{1}$ Ticiana Leal, M.D., ${ }^{2.6}$ Darya Buehler, M.D., ${ }^{2,7}$ Gopal lyer, Ph.D., ${ }^{1,2}$ Jacob G. Scott, M.D., D.Phil., ${ }^{8}$ Randall J. Kimple, M.D., Ph.D., ${ }^{1,2}$

1. Department of Human Oncology, School of Medicine and Public Health, University of Wisconsin, Madison, WI, USA

2. University of Wisconsin Carbone Cancer Center, School of Medicine and Public Health, University of Wisconsin, Madison, WI, USA

3. Case Western Reserve University, Cleveland, OH 44195, USA

4. Department of Biostatistics and Medical Informatics, University of Wisconsin School of Medicine and Public Health, University of Wisconsin, Madison, WI, USA

5. Department of Obstetrics and Gynecology, University of Wisconsin School of Medicine and Public Health, University of Wisconsin, Madison, WI, USA

6. Department of Medicine, Division of Hematology/Oncology, School of Medicine and Public Health, University of Wisconsin, Madison, WI, USA

7. Department of Pathology and Laboratory Medicine, University of Wisconsin School of Medicine and Public Health, University of Wisconsin, Madison, WI, USA

8. Department of Radiation Oncology, Taussig Cancer Institute, Cleveland Clinic, 10201 Carnegie Ave, Cleveland, OH 44195, USA

\section{Corresponding Authors:}

Andrew M. Baschnagel, MD

Department of Human Oncology

University of Wisconsin-Madison School of Medicine and Public Health

600 Highland Avenue, K4/B100-0600

Madison, WI 53792

Phone: 608-263-8500

Email: baschnagel@humonc.wisc.edu 
Randall J. Kimple, MD, PhD

Department of Human Oncology

University of Wisconsin-Madison School of Medicine and Public Health

3107 WIMR, 1111 Highland Ave., Madison, WI 53705,

Phone: (608) 263-8500,

Email: rkimple@humonc.wisc.edu

Running Title: NSCLC patient-derived xenografts

Keywords: non-small cell lung cancer, brain metastasis, patient-derived xenografts

Disclosures: none

Funding: This project was supported in part by grants from the American Cancer Society (RSG-16-091-01-TBG), the UW Paul P. Carbone Young Investigator Award, the University of Wisconsin Lung Disease Oriented Team, and the University of Wisconsin Carbone Cancer Center Support Grant (P30 CA014520). 


\section{Abstract}

\section{Introduction:}

The purpose of this study was to establish and characterize a direct-from patient-derived xenograft (PDX) model of non-small cell lung cancer (NSCLC) brain metastases.

\section{Methods:}

Surgically obtained tissue was implanted subcutaneously and as orthotopic intracranial implants into immunodeficient mice. Histology and DNA loci were compared between original tumor and subsequent PDX passages. Tumors underwent RNA and DNA sequencing and relevant therapeutic targets were identified. Tumor growth rates were assessed following treatment with radiation, MEK inhibitor selumetinib, or MET inhibitor savolitinib. Cell lines were established.

\section{Results:}

Nine NSCLC brain metastases PDXs were established. Morphologically, strong retention of cytoarchitectural features was observed between original patient tumor and subcutaneous and intracranial tumors. Short tandem repeat analysis demonstrated strong concordance between patient tumors and subsequent PDX passages. Transcriptome and mutation analysis revealed high correlation between matched patient and PDX samples. Significant growth inhibition occurred with radiation, with selumetinib in tumors harboring KRAS G12C mutations and with savolitinib in a tumor with MET exon 14 skipping mutation. The combination of radiation and savolitinib resulted in significant tumor growth delay compared to radiation or savolitinib alone our MET exon 14 skipping mutation PDX. Early passage cell strains showed high consistency between patient and PDX tumors.

\section{Conclusion:}

We have established a robust human xenograft model system for investigating NSCLC brain metastases. These PDXs and cell lines show strong phenotypic and molecular correlation with the original patient tumors and provide a valuable resource for testing preclinical therapeutics. 


\section{Introduction}

Brain metastases from non-small cell lung cancer (NSCLC) are unfortunately a common event that occur in more than a quarter of patients with stage IV disease at diagnosis ${ }^{1}$, and up to $30 \%$ of patients developing central nervous system (CNS) progression during the course of their disease. $^{1-3}$ Once diagnosed, the prognosis remains poor, with a median survival of 9-15 months. ${ }^{4}$ Treatment options are evolving as patients treated with systemic therapies have better control of their extracranial disease and live longer, which allows time for brain metastases to develop.

Standard treatments for brain metastases are surgical resection and stereotactic radiosurgery for limited lesions or whole brain irradiation for multiple metastases. ${ }^{5}$ Whole brain irradiation has significant toxicity ${ }^{5}$ and cytotoxic chemotherapy has a limited role due to its inability to cross the blood-brain barrier. ${ }^{5,6}$ A proportion of patients with brain metastases harboring alterations in $E G F R$ or $A L K$ can achieve intracranial control with small molecule tyrosine kinase inhibitors ${ }^{7,8}$; however most patients with NSCLC do not harbor these genomic alterations. In a phase II study, patients with NSCLC and brain metastases with PD-L1 expression of at least $1 \%^{9}$ benefited from PD-1 blockade, with CNS responses (30\%) similar to extracranial responses, indicating that immunotherapy can results in prolonged survival in a subset of patients. However, these response rates are far below those seen following standard of care radiotherapy. Overall, there is a need to develop therapies that have better intracranial efficacy with less toxicity.

One barrier to improving the therapeutic approach to brain metastases is the lack of robust preclinical brain metastasis models. To our knowledge, there are currently no commercial NSCLC brain metastasis cell lines available and in vivo brain metastasis models are not widely accessible. Patient-derived xenografts (PDXs) are a well-established model to study cancers in the preclinical setting. ${ }^{10}$ PDXs are established by obtaining tumor samples directly from patients and subsequently implanting and passaging these tumors in immunodeficient mice. ${ }^{10}$ PDXs can be grown in the flank or hind leg of mice or as orthotopic brain implants. For brain tumors, orthotopic intracranial implants allow cancer cells to more closely resemble their original patient tumors both phenotypically and genotypically given the influence of the brain microenvironment. ${ }^{11}$

Here, we report on the development and characterization of a cohort of NSCLC brain metastasis PDX models, including both flank and orthotopic intracranial xenografts as well as 
two NSCLC brain metastasis-derived cell lines. We demonstrate the utility of these PDXs by assessing their response to radiation and selumetinib in KRAS mutated xenografts and savolitinib in a MET exon 14 mutated xenograft.

\section{Materials and Methods}

\section{Patient Selection}

Patients with newly diagnosed or recurrent NSCLC brain metastases, undergoing surgical resection, were consented to participate in this IRB-approved protocol.

\section{Mice}

Immunodeficient NOD SCID gamma (NSG) (Envigo, Indianapolis) and Hsd:athymic NudeFoxn1nu (Envigo, Indianapolis) mice were used for PDX development. All mice were kept in the Association for Assessment and Accreditation of Laboratory Animal Care approved Wisconsin Institute for Medical Research Animal Care Facility, and studies were carried out in accordance with an approved animal protocol.

\section{DNA Sequencing}

After expert pathologist assessment, solid tumor genomic DNA was isolated from formalin-fixed, paraffin-embedded tumor samples. DNA libraries were prepared using the KAPA Hyper Prep Kit, hybridized to the xT probe set, and amplified with the KAPA HiFi HotStart ReadyMix. The amplified target-captured DNA tumor libraries were sequenced to an average target depth of 500x on an Illumina HiSeq 4000 using the Tempus xT (Tempus labs Chicago, IL, USA) gene panel. The panel analyzes single nucleotide variants (SNVs), indels, and copy number variants in 596 genes and genomic rearrangements in 21 genes with an average coverage of 500x. ${ }^{12}$ Variants were called using Freebayes (version 1.0.2). Clinically relevant mutations were identified using the Cancer Genome Interpretor ${ }^{13}$ and ClinVar $^{14}$.

\section{Establishment of Xenografts}

Patient tissue collected during surgery was added to a $1.5 \mathrm{ml}$ tube with media (Dulbecco's Modified Eagle Medium with 10\% fetal bovine serum, $1 \%$ penicillin/streptomycin, and $2.5 \mu \mathrm{g} / \mathrm{mL}$ amphotericin B) and minced with sharp, sterile scissors into a slurry. Matrigel (\#354230, BD Biosciences, Inc) was added to the media in a 1:1 ratio and injected subcutaneously into NSG mice with an 18 gauge needle. Subsequent passages were made in a similar fashion into either NSG or athymic nude mice. Cryopreservation of xenografts was accomplished by mixing 
minced tumor pieces with complete transport media supplemented with $10 \%$ dimethylsulfoxide (DMSO) as previously described. ${ }^{15-18}$ Tumors were frozen in controlled rate freezers to $-80^{\circ} \mathrm{C}$ overnight and transferred to liquid nitrogen for long-term storage. To thaw tumors, aliquots were warmed to $37^{\circ} \mathrm{C}$ in a heated water bath and tumor tissue was washed twice in transport complete media (without DMSO) and immediately implanted into mice.

\section{Establishment of Cell Lines}

Tumors taken from early PDX passages were dissociated into single cell suspensions and seeded in T-25 flasks in media (Dulbecco's Modified Eagle Medium with 10\% fetal bovine serum, $1 \%$ penicillin/streptomycin, and $2.5 \mu \mathrm{g} / \mathrm{mL}$ amphotericin B) and incubated at $37^{\circ} \mathrm{C}$ in a humidified atmosphere of $5 \% \mathrm{CO} 2$. Media was changed regularly, and cells were passaged once they reached $70 \%$ confluence. Mycoplasma testing and short tandem repeat profiling was performed during passaging.

\section{Short Tandem Repeat (STR) Profiling}

DNA typing by multiplex polymerase chain reaction (PCR) of short repetitive elements of highly polymorphic markers was performed to assess genetic stability of xenografts and cell lines. STR profiling and analysis was performed using the PowerPlex® 16 HS System (Promega, Madison WI).

\section{Orthotopic Intracranial Implants}

Tissue was enzymatically dissociated to single cells and $2 \times 10^{5}$ cells were suspended in $5 \mu$ of phosphate-buffered saline. Using a Hamilton syringe, the cells were stereotactically injected under sterile conditions into the right striatum of anesthetized NOD SCID mice at $1 \mu \mathrm{L} / \mathrm{min}$ at the following coordinates referenced from bregma: $0 \mathrm{~mm}$ antero-posterior, $+2.5 \mathrm{~mm}$ medio-lateral, and $-3.5 \mathrm{~mm}$ dorso-ventral. The scalp was closed with 6-0 polypropylene suture. Mice were placed on a heating pad in sterile cages and allowed to awaken from anesthesia. One week after injection animals were placed onto a small-animal MRI scanner (4.7-T horizontal bore imaging/spectroscopy system; Varian, Palo Alto, CA), and T1- and T2-weighted images were obtained. As per animal protocol, once neurological symptoms were observed, mice were immediately euthanized by perfusion fixation with 10\% paraformaldehyde and moved to $70 \%$ ethanol solution after 48 hours. Brains were then excised, formalin fixed, embedded in paraffin, and processed for histology.

Immunohistochemistry 
At each passage, tumor was fixed in 10\% neutral-buffered formalin and embedded in paraffin blocks. Five- $\mu \mathrm{m}$ sections of PDX and matching patient tumors were stained with hematoxylin and eosin (H\&E) and imaged on an Olympus BX51 microscope (Olympus America, Inc). TTF-1 (clone SPT24, BioCare, 1:100) staining was performed with a BenchMark Ultra IHC/ISH System (Roche Diagnostics, Rotkreuz, Switzerland); according to manufactures established protocol. Additional Immunohistochemistry (IHC) details and antibodies used can be found in the supplemental materials.

\section{RNA Sequencing}

Total RNA was isolated by using the QIAGEN AllPrep Kit. RNA sequencing libraries were prepared using the KAPA Stranded RNA-Seq Kit with RiboErase (HMR; KAPA Biosystems). RNA was fragmented to a size of 100-200 nucleotides and amplified for 11 cycles. Final libraries were quantified on a high-sensitivity bioanalyzer chip and sequenced on the NextSeq 550 (Illumina).

\section{Gene Expression and Pathway Analysis}

RNA-Seq data were aligned to human reference sequence GRCh38 using RSEM ${ }^{19}$, and expression quantification per gene was computed. Raw read counts were normalized by the trimmed mean of $M$-values method ${ }^{20}$. Differential gene expression (DE) analysis was performed using a likelihood ratio test with the $R$ package edge $R^{21,22}$. The $p$-values were adjusted to control for false discoveries using the Benjamini-Hochberg method ${ }^{23}$. Principal component analysis (PCA) was performed on log2 transformed count per million values. The gene lists were sorted as increasing or decreasing. For the increasing rankings, the genes with positive log fold change (logFC) were sorted by q-value in increasing order, followed by genes with negative logFC sorted by q-value in decreasing order. For the decreasing rankings, the increasing list was reversed.

Gene ontology (GO) and gene set enrichment analyses (GSEA) were performed using goseq ${ }^{24}$ and GSEAPreranked ${ }^{25}$, and p-values were adjusted with Benjamini-Hochberg method. The GOseq analysis was performed using the annotation package Bioconductor TxDb.Hsapiens.UCSC.hg38.knownGene: Annotation package for TxDb object(s). (Team BC, Maintainer BP (2019), R package version 3.4.6.). GSEAPreranked was performed using the KEGG subset of the canonical pathways gene sets from MSigDB version $7^{26,27}$. For identifying annotations overrepresented in the supervised approach, the top 1\% (219) of the genes were extracted from the DE increasing rankings; in the unsupervised approach, the top 1\% (219 
genes) were extracted from the PC2 increasing rankings. For identifying annotations overrepresented in the patient tumors increasing rankings were used and for PDX samples decreasing rankings were used. To mitigate the high false discovery rate with GSEAPreranked $^{28}$, a filtering step as described by Yoon et al. $^{28}$ was implemented. Pearson correlation coefficient of the log2 transformed gene expression values was computed, first using all of the genes for each pair of samples and then excluding the top $1 \%$ of genes from the PC2 increasing rankings.

\section{Mutation calling from RNA-Seq}

Bulk RNA-Seq files are pre-processed for quality control using fastp with default parameters ${ }^{29}$. STAR aligner in two-pass mode is used to align the reads to GRCh38 and GRCm38 genomes ${ }^{30}$. Aligned bam files for PDX data are further filtered to remove reads aligning to the host genome with high confidence using $\mathrm{R}$ package Xenofilte $^{31}$. Following read splitting and duplicate removal, we have utilized Strelka2 in rna mode to call variants with default parameters ${ }^{32}$. Variants passing the default filters and read depth 20 are annotated using Ensembl VEP and filtered to include only high impact variants for downstream analysis.

\section{Tumor Growth Delay Experiments}

Tumor cells $\left(5 \times 10^{6}\right)$ suspended in saline were injected subcutaneously into the hind leg. When tumors grew to a mean volume of $150 \mathrm{~mm}^{3}$, mice were randomized into control group, radiation alone group (2 Gy per fractions in 1-2 weeks), selumitinib group (25 mg/kg BID for 2 weeks), savolitinib group $(2.5 \mathrm{mg} / \mathrm{kg}$ for 2 weeks) or radiation plus savolitinib group $(2.5 \mathrm{mg} / \mathrm{kg}$ for 2 weeks). Selumitinib and savolitinib were administered by PO gavage. Each experimental and control group contained 10 mice. Tumor volume was measured twice weekly with Vernier calipers and calculated using: $V=\left(\frac{\pi}{6}\right) x$ (large diameter) $x$ (small diamter) ${ }^{2}$. Tumors were followed until the group reached a mean size of 1,500-2,000 $\mathrm{mm}^{3}$. Specific tumor growth delay was calculated as the number of days for the mean of the treated tumors to grow to $500 \mathrm{~mm}^{3}$ minus the number of days for the mean of the control group to reach the same size. The percentage of tumor growth inhibition $(\% \mathrm{TGI}=1-$ [changes of tumor volume in treatment group/changes of tumor volume in control group] $\times 100$ ) was used for the evaluation of anti-tumor efficacy. The radiation dose enhancement factor was calculated by dividing the normalized tumor growth delay in mice treated with both savolitinib and radiation by the absolute growth delay in mice treated with radiation alone. Normalized tumor growth was 
defined as the time in days for tumors to reach $1,000 \mathrm{~mm}^{3}$ in mice treated with the combination of savolitinib and radiation minus the time in days in mice treated with savolitinib alone.

\section{Statistical Analysis}

Differences between tumor mean values was determined using a Student's t-test. Data are presented as the mean \pm standard error of the mean (SEM). A probability level of a $p$ value of $<0.05$ was considered significant. All graphs and analyses were performed and graphed using GraphPad Prism version 8.0 (GraphPad Software, San Diego, CA).

\section{Results}

\section{Patient Characteristics}

Fourteen patients with NSCLC brain metastases were consented for tumor collection and establishment of xenografts. Overall, nine brain metastasis samples have given rise to viable xenograft tumors with a take rate of $64 \%$. Clinical characteristic of the patient and their tumors that successfully formed xenografts are shown in Figure 1. The average age of the donors was 61 (range 38-84). All tumors were adenocarcinomas except one, which was a sarcomatoid carcinoma with an adenocarcinoma (TTF-1 positive) component. The majority $(71 \%)$ were diagnosed as synchronous brain metastases. Two patients underwent surgical resection for salvage treatment after progression following whole brain irradiation.

All nine patient brain metastasis samples underwent amplicon DNA sequencing (Figure 1). The most common genomic alterations were TP53 mutations in six tumors and KRAS mutations in five tumors. Other relevant genomic alterations include $M Y C$ amplification and loss of $C D K N 2 A$. One tumor also harbored a MET exon 14 splice site mutation. Programmed death-ligand 1 (PDL1) expression of the patient brain metastasis tumors ranged from $<1 \%$ to $100 \%$ (Figure 1 and supplemental Table 1).

\section{Establishment and Characterization of Xenografts and Cell Lines}

All nine PDX tumors were passaged at least 5 times as subcutaneous xenografts with a mean time between passages of 46.4 days (22-114 days). There were no differences between time of passage from initial tumor implantation to first passage versus fourth to fifth passage $(p=0.52)$. Morphologically, strong retention of cytoarchitectural features was observed between patient tumor and subcutaneous PDX. Figure 2 shows representative H\&E images of the patient sample and PDX passage 1 (P1) and passage 5 (P5). TTF-1 expression status was also highly consistent between patient tumor and PDX (Figure 2). Genetic profiles of the PDXs were 
confirmed with STR analysis (Supplemental Table 2). All PDXs showed 89-100\% allelic match to the original patient samples. PDXs continued to retain their STR profiles from passage one to passage five (Supplemental Table 2). Comprehensive tumor growth rate assessment was carried out for five PDXs (Figure 3A). Orthotopic intracranial models were successfully generated from two tumor samples; University of Wisconsin (UW)-lung-2 and UW-lung-16. MRI images shows the formation of brain metastasis and adjacent H\&E confirmed tumor which was morphologically similar to the patient and flank tumors (Figure 3B). STR testing confirmed 100\% and $92.8 \%$ matching of alleles for UW-lung-2 and UW-lung-16 orthotopic tumors, respectively. Cell lines were successfully established from two PDXs, UW-lung-2 and UW-lung-16, following at least 15 in vitro passages (Figure 3C). STR testing of subsequent passages confirmed that these lines retained their genetic profile (supplemental table 2).

\section{Transcriptome Analysis of patient and PDXs tumors}

RNA-Seq analysis was performed on four patient and corresponding flank PDX samples. One PDX (UW-lung-16) was sequenced twice and was used as a control replicate. Mouse-specific reads were filtered in silico from the PDX RNA samples before aligning reads to the human genome. Overall, patient and PDX samples highly correlated with each other (Figure 4A). The average Pearson correlation coefficient for case-matched tumors was 0.88 and ranged from 0.86 to 0.90 . Using a supervised analysis comparing matched patient tumors and PDXs, there were 21 Kyoto Encyclopedia of Genes and Genomes (KEGG) pathways that were significantly over represented in patient tumor samples $(q<0.001)$ and 12 processes that were significantly over represented in the PDX tumors ( $q<0.001$ ) (Supplemental Table 3). There were 231 Gene Ontology (GO) annotations overrepresented in patients $(q<0.001)$ and $185 \mathrm{GO}$ annotations significantly overrepresented in PDX tumors (Supplemental Table 4). The top 10 most significantly overrepresented GO annotations in the patient tumor samples consisted of cellular processes associated with immune regulation while the top pathways overrepresented in the PDXs included those related to cell cycle and DNA replication (Figure 4B).

PCA of matched patient and PDX tumor gene expression revealed distinct clustering of PDXs compared to patient tumor samples when viewed through PC1 and PC2 (Figure 4C-E). PC2 appeared to be the main contributor of the differences between patient and PDX samples (Figure 4C). By contrast, viewing the PCA through PC1/PC3 and PC1/PC4 revealed close clustering of matched PDX samples to their corresponding patient sample. (Figure 4D and 4E). On Gene Ontology enrichment analysis, PC2 is highly represented by immune cell processes 
(Figure 4F). PC1, PC3 and PC4 have little representation of immune processes and appear to represent differences in intrinsic tumor biology (Supplemental Table 4).

Given the differences seen in PC2, we hypothesized that PDXs would associate most similarly to their matched patient tumors compared with unmatched tumors after controlling for the differences in immune cell populations. We performed an analysis removing the top $1 \%$ of genes contributing to PC2. This removed 219 genes that were the most prevalent genes associated with immune cell populations (Figure 4F), and a stronger transcriptional resemblance between patient and PDX tumor pairs was seen. The average Pearson correlation coefficient for case-matched tumors and PDXs increased from 0.88 to 0.90 (Figure 4G).

Patient and PDX Variant Allele Fraction Analysis (VAF)

Variants detected by RNA-Seq for UW-lung-2, UW-lung-4, UW-lung-5 and UW-lung-16 were compared between patient tumors and PDXs grown in the brain and in the flank. High correlations were observed between samples with Pearson correlation coefficients ranging between 0.794 and 0.839 (Figure 5A and B). For UW-lung-2 a comparison between patient brain metastasis and PDX grown in the flank and PDX grown in the brain was also performed. Overall, there were 1,979 alterations detected in the UW-lung-2 patient brain metastasis with $96.5 \%$ of these alterations identified in the PDX samples (Figure $5 \mathrm{~A}$ ). There was a higher correlation in VAF distribution $\left(R^{2}=0.848\right.$ and 0.794 , respectively) of shared variants, between the patient and the PDX brain metastasis compared to patient and PDX flank tumors. Overall, these findings indicate that the mutation landscape is conserved between patient and PDX samples. In addition, our orthotopic intracranial PDX more closely resembles the originating patient brain metastasis compared to the heterotopic PDX.

\section{PDX Response to Radiotherapy}

Given that radiation is the predominate therapeutic modality for the treatment of brain metastases, five PDXs were tested for response to standard fractionated radiotherapy (Figure $6 A)$. All tumors responded to radiation with tumor growth inhibition values at the end of the experiments of $51 \%$ for UW-lung-2, $42 \%$ for UW-lung-5, 53\% for UW-lung- $16,58 \%$ for UW-lung18 and $92 \%$ for UW-lung-21 ( $P<0.01$ for all).

\section{PDX Response to MEK Inhibitor Selumetinib}

$K R A S$ is one of the most commonly mutated genes in lung adenocarcinoma ${ }^{33}$, and in our cohort five PDX tumors harbored KRAS mutations. Mutations in KRAS leads to activation of the 
mitogen-activated protein kinase (MAPK) pathway involving MAPK kinase (MEK). As an initial effort to assess the utility of these PDXs for therapeutic response to molecular targeted therapy, we selected two tumors harboring KRAS G12C mutations and tested their response to the MEK inhibitor selumetinib. Both tumors responded to selumetinib; however, tumor growth inhibition was only significant in UW-lung-2 $(p=0.005)$ and not significant in the UW-lung $16(p=0.34)$ (Figure 6B). IHC analysis revealed a decrease in phospho-MAPK, pS6 and Ki67 with treatment of selumetinib at 24 hours in UW-lung-2. In UW-lung-16, there was minimal change in phosphoMAPK and Ki-67, but a decrease in pS6 (Figure 6C) with selumetinib treatment at 24 hours.

\section{PDX Response to MET Inhibitor Savolitinib}

MET exon 14 skipping mutations occur in 3-4\% of lung adenocarcinomas and results in the deletion of the intracellular juxtamembrane domain of the receptor, leading to enhanced signaling and tumor proliferation. ${ }^{33,34}$ Small molecule inhibitors have shown therapeutic efficacy in NSCLC harboring MET exon 14 mutations. ${ }^{34}$ We therefore tested the therapeutic response of UW-lung-21, which harbors a MET exon 14 mutation, to the selective MET inhibitor savolitinib. Savolitinib alone significantly inhibited tumor growth in UW-lung-21 with a tumor growth inhibition value of $46 \%(p<0.01$, Figure $6 \mathrm{D})$. We next tested the capacity of savolitinib to augment the response of radiation. Previous studies have shown mixed results when combining radiation with MET inhibitors. ${ }^{35-37}$ The combination of savolitinib and radiation significantly delayed the growth compared to vehicle control, savolitinib or radiation alone $(p<0.01$; Figure $6 D)$. The mean time for tumors to reach a size of $1,000 \mathrm{~mm}^{3}$ was 12.3 days for vehicle-treated mice; 21.6 days for savolitinib-treated mice, 40.5 days for irradiated mice and 54.7 days in mice that received savolitinib plus irradiation $(P<0.001)$. The absolute growth delay was 9.3 days for savolitinib alone, 28.2 days for irradiation alone and 42.4 days for savolitinib plus irradiation. The radiation dose enhancement factor for savolitinib was 1.2, indicating that savolitinib enhances radiation-induced tumor growth delay. IHC performed on UW-lung-21-treated tumors demonstrated inhibition of phospho-MET and pS6 and decrease in Ki67 with savolitinib treatment (Figure 6E).

\section{Discussion}

Brain metastases from NSCLC frequently occur, remain difficult to treat and if not controlled can lead to significant neurologic compromise and ultimately death. ${ }^{2}$ Current standard approaches involving surgery and/or whole brain radiotherapy, while effective, can significantly impair quality of life. Stereotactic radiosurgery can provide excellent control but continued development of new 
brain metastases remains a challenge. ${ }^{2,5}$ Despite molecular-targeted agents that penetrate the blood-brain barrier, disease progression in the brain remains a problem. The lack of preclinical NSCLC brain metastasis models is one barrier that may be preventing the development of effective therapeutic strategies.

Here we describe the development of a panel of NSCLC brain metastasis PDXs and demonstrate their preclinical utility in evaluating therapies. We accomplished a high engraftment efficiency, and showed that these PDXs closely resemble the patient's original metastatic tumor by retaining their morphologic and molecular features. In addition, we demonstrated the capability of these tumors to grow as cell lines and as intracranial implants making them a valuable resource to study brain metastases. To expand upon their translational potential, we characterized the PDXs response to radiotherapy, demonstrated tumor growth inhibition with MEK inhibition in KRAS mutated PDXs and showed significant growth delay with the MET inhibitor savolitinib in a PDX harboring a MET exon 14 mutation. Furthermore, combining savolitinib with clinically meaningful doses of radiation resulted in radiosensitization in our MET exon 14 mutated NSCLC PDX. This combination provides a potential treatment strategy for patients with brain metastases from exon 14 mutated NSCLC.

PDXs have both advantages and disadvantages compared to other NSCLC brain metastasis models. PDXs allow for quick testing of molecular-targeted therapies, recapitulate human tumors and are cost effective. Other available models include human and murine cell line xenografts, spontaneous murine models, and humanized mouse xenografts.

Human cell line models generally show a homogeneous histology and lack human stroma, which is important for modeling the metastatic process. These cell lines are derived by serially passaging cells in culture, inoculating them in immunodeficient mice and selecting cells that seed the brain. There are two known available NSCLC cell lines that have tropism for the brain, H2030-BrM3 and PC9-BrM3, both established from lymph nodes of patients with lung adenocarcinoma and not from brain metastasis tumors. ${ }^{38}$ Syngeneic murine cell line models allow for assessment of immunotherapies but are dependent on mouse tumors cell lines, which are typically driven by specific driver mutations and may not reliably recapitulate human disease. The only reported syngeneic murine cell line model used Lewis lung carcinoma cells either directly implanted into the brain or forming brain tumors through hematogenous spread by intracardiac injections. ${ }^{39}$ 
Spontaneous brain metastases can be modeled in immunocompetent, genetically engineered mice (GEM) by manipulating oncogenes or tumor suppressor genes. ${ }^{40,41}$ Currently there are no available NSCLC brain metastasis GEM models; however, two models of melanoma ${ }^{42,43}$ and one model of small cell lung cancer ${ }^{44}$ have been reported. In these models, the formation of metastases is limited because primary tumors grow quickly and animals usually have to be euthanized before brain metastases develop. ${ }^{45}$

Compared to cell line and GEM models, PDXs are directly established from patients and thus closely reproduce the molecular, genetic and histological heterogeneity of the original tumor. The disadvantage of PDXs is the lack of an immune system - however, this can be overcome by using humanized mouse PDX models. Humanized mouse models engraft components of the human immune system into otherwise immunocompromised mice. ${ }^{46}$ Ideally, humanized mice would have the same immune system from which the PDX was derived. However, it is difficult to obtain CD34+ cells from cancer patients, and therefore, an allogeneic immune approach is typically used. ${ }^{47}$ These are complex models and currently no humanized mouse models for NSCLC brain metastasis exist.

In conclusion, we have developed a panel of human derived NSCLC brain metastasis xenografts and cell lines, which have the capability of forming intracranial tumors. These PDXs display a spectrum of differentiation typical of clinical pulmonary adenocarcinoma samples, and a high degree of phenotypic and genetic consistency between original patient tumor and xenograft. The use of early passage xenografts and cell strains provides a powerful system for investigating novel therapeutics and combination therapies, and for testing hypotheses of mechanisms of therapeutic response and resistance in human NSCLC brain metastases. 


\section{Acknowledgements:}

We would like to thank Dr. M. Shahriar Salamat and University of Wisconsin Department of Pathology and Laboratory Medicine for initial tissue processing of patient samples. We also would like to acknowledge the University of Wisconsin Carbone Cancer Center Translational Science BioCore Biobank for providing tumor samples for PDX development.

\section{References}

1. Waqar SN, Samson PP, Robinson CG, et al. Non-small-cell Lung Cancer With Brain Metastasis at Presentation. Clin Lung Cancer. 2018;19(4):e373-e379.

2. Langer CJ, Mehta MP. Current management of brain metastases, with a focus on systemic options. Journal of clinical oncology : official journal of the American Society of Clinical Oncology. 2005;23(25):6207-6219.

3. Sun A, Hu C, Wong SJ, et al. Prophylactic Cranial Irradiation vs Observation in Patients With Locally Advanced Non-Small Cell Lung Cancer: A Long-term Update of the NRG Oncology/RTOG 0214 Phase 3 Randomized Clinical Trial. JAMA Oncol. 2019;5(6):847-855.

4. Sperduto PW, Yang TJ, Beal K, et al. Estimating Survival in Patients With Lung Cancer and Brain Metastases: An Update of the Graded Prognostic Assessment for Lung Cancer Using Molecular Markers (Lung-molGPA). JAMA Oncol. 2017;3(6):827-831.

5. Baschnagel A, Wolters PL, Camphausen K. Neuropsychological testing and biomarkers in the management of brain metastases. Radiation oncology. 2008;3:26.

6. Deeken JF, Loscher W. The blood-brain barrier and cancer: transporters, treatment, and Trojan horses. Clin Cancer Res. 2007;13(6):1663-1674.

7. Soria JC, Ohe Y, Vansteenkiste J, et al. Osimertinib in Untreated EGFR-Mutated Advanced NonSmall-Cell Lung Cancer. N Engl J Med. 2018;378(2):113-125.

8. Peters S, Camidge DR, Shaw AT, et al. Alectinib versus Crizotinib in Untreated ALK-Positive NonSmall-Cell Lung Cancer. N Engl J Med. 2017;377(9):829-838.

9. Goldberg SB, Schalper KA, Gettinger SN, et al. Pembrolizumab for management of patients with NSCLC and brain metastases: long-term results and biomarker analysis from a non-randomised, open-label, phase 2 trial. Lancet Oncol. 2020.

10. Tentler JJ, Tan AC, Weekes CD, et al. Patient-derived tumour xenografts as models for oncology drug development. Nat Rev Clin Oncol. 2012;9(6):338-350.

11. Camphausen $\mathrm{K}$, Purow $B$, Sproull $M$, et al. Influence of in vivo growth on human glioma cell line gene expression: convergent profiles under orthotopic conditions. Proc Natl Acad Sci U S A. 2005;102(23):8287-8292.

12. Beaubier N, Tell R, Lau D, et al. Clinical validation of the tempus $x T$ next-generation targeted oncology sequencing assay. Oncotarget. 2019;10(24):2384-2396.

13. Tamborero D, Rubio-Perez C, Deu-Pons J, et al. Cancer Genome Interpreter annotates the biological and clinical relevance of tumor alterations. Genome Med. 2018;10(1):25.

14. Landrum MJ, Lee JM, Benson M, et al. ClinVar: improving access to variant interpretations and supporting evidence. Nucleic Acids Res. 2018;46(D1):D1062-D1067.

15. Kimple RJ, Harari PM, Torres AD, et al. Development and characterization of HPV-positive and HPV-negative head and neck squamous cell carcinoma tumorgrafts. Clin Cancer Res. 2013;19(4):855-864. 
16. Stein AP, Saha S, Liu CZ, Hartig GK, Lambert PF, Kimple RJ. Influence of handling conditions on the establishment and propagation of head and neck cancer patient derived xenografts. PLoS One. 2014;9(6):e100995.

17. Swick AD, Stein AP, McCulloch TM, et al. Defining the boundaries and expanding the utility of head and neck cancer patient derived xenografts. Oral Oncol. 2017;64:65-72.

18. Stein AP, Swick AD, Smith MA, et al. Xenograft assessment of predictive biomarkers for standard head and neck cancer therapies. Cancer Med. 2015;4(5):699-712.

19. Li B, Dewey CN. RSEM: accurate transcript quantification from RNA-Seq data with or without a reference genome. BMC Bioinformatics. 2011;12:323.

20. Robinson MD, Oshlack A. A scaling normalization method for differential expression analysis of RNA-seq data. Genome Biol. 2010;11(3):R25.

21. McCarthy DJ, Chen Y, Smyth GK. Differential expression analysis of multifactor RNA-Seq experiments with respect to biological variation. Nucleic Acids Res. 2012;40(10):4288-4297.

22. Robinson MD, McCarthy DJ, Smyth GK. edgeR: a Bioconductor package for differential expression analysis of digital gene expression data. Bioinformatics. 2010;26(1):139-140.

23. Benjamini Y, Hochberg Y. Controlling the False Discovery Rate: A Practical and Powerful Approach to Multiple Testing. Journal of the Royal Statistical Society Series B (Methodological). 1995;57(1):289-300.

24. Young MD, Wakefield MJ, Smyth GK, Oshlack A. Gene ontology analysis for RNA-seq: accounting for selection bias. Genome Biol. 2010;11(2):R14.

25. Subramanian A, Tamayo P, Mootha VK, et al. Gene set enrichment analysis: a knowledge-based approach for interpreting genome-wide expression profiles. Proc Natl Acad Sci U S A. 2005;102(43):15545-15550.

26. Kanehisa M, Goto S. KEGG: kyoto encyclopedia of genes and genomes. Nucleic Acids Res. 2000;28(1):27-30.

27. Liberzon A, Subramanian A, Pinchback R, Thorvaldsdottir H, Tamayo P, Mesirov JP. Molecular signatures database (MSigDB) 3.0. Bioinformatics. 2011;27(12):1739-1740.

28. Yoon S, Kim SY, Nam D. Improving Gene-Set Enrichment Analysis of RNA-Seq Data with Small Replicates. PLoS One. 2016;11(11):e0165919.

29. Chen S, Zhou Y, Chen Y, Gu J. fastp: an ultra-fast all-in-one FASTQ preprocessor. Bioinformatics. 2018;34(17):i884-i890.

30. Dobin A, Davis CA, Schlesinger F, et al. STAR: ultrafast universal RNA-seq aligner. Bioinformatics. 2013;29(1):15-21.

31. Kluin RJC, Kemper K, Kuilman T, et al. XenofilteR: computational deconvolution of mouse and human reads in tumor xenograft sequence data. BMC Bioinformatics. 2018;19(1):366.

32. Kim S, Scheffler K, Halpern AL, et al. Strelka2: fast and accurate calling of germline and somatic variants. Nat Methods. 2018;15(8):591-594.

33. Cancer Genome Atlas Research N. Comprehensive molecular profiling of lung adenocarcinoma. Nature. 2014;511(7511):543-550.

34. Awad MM, Oxnard GR, Jackman DM, et al. MET Exon 14 Mutations in Non-Small-Cell Lung Cancer Are Associated With Advanced Age and Stage-Dependent MET Genomic Amplification and c-Met Overexpression. Journal of clinical oncology : official journal of the American Society of Clinical Oncology. 2016;34(7):721-730.

35. Baschnagel AM, Galoforo S, Thibodeau BJ, et al. Crizotinib Fails to Enhance the Effect of Radiation in Head and Neck Squamous Cell Carcinoma Xenografts. Anticancer Res.

2015;35(11):5973-5982. 
36. Bhardwaj V, Zhan Y, Cortez MA, et al. C-Met inhibitor MK-8003 radiosensitizes c-Met-expressing non-small-cell lung cancer cells with radiation-induced c-Met-expression. $J$ Thorac Oncol. 2012;7(8):1211-1217.

37. Tumati V, Kumar S, Yu L, Chen B, Choy H, Saha D. Effect of PF-02341066 and radiation on nonsmall cell lung cancer cells. Oncol Rep. 2013;29(3):1094-1100.

38. Nguyen DX, Chiang AC, Zhang XH, et al. WNT/TCF signaling through LEF1 and HOXB9 mediates lung adenocarcinoma metastasis. Cell. 2009;138(1):51-62.

39. Zhang Z, Hatori T, Nonaka H. An experimental model of brain metastasis of lung carcinoma. Neuropathology. 2008;28(1):24-28.

40. Heyer J, Kwong LN, Lowe SW, Chin L. Non-germline genetically engineered mouse models for translational cancer research. Nat Rev Cancer. 2010;10(7):470-480.

41. Sharpless NE, Depinho RA. The mighty mouse: genetically engineered mouse models in cancer drug development. Nat Rev Drug Discov. 2006;5(9):741-754.

42. Cho JH, Robinson JP, Arave RA, et al. AKT1 Activation Promotes Development of Melanoma Metastases. Cell Rep. 2015;13(5):898-905.

43. Kato $\mathrm{M}$, Takahashi $\mathrm{M}$, Akhand $\mathrm{AA}$, et al. Transgenic mouse model for skin malignant melanoma. Oncogene. 1998;17(14):1885-1888.

44. Meuwissen R, Linn SC, Linnoila RI, Zevenhoven J, Mooi WJ, Berns A. Induction of small cell lung cancer by somatic inactivation of both Trp53 and Rb1 in a conditional mouse model. Cancer Cell. 2003;4(3):181-189.

45. Gomez-Cuadrado L, Tracey N, Ma R, Qian B, Brunton VG. Mouse models of metastasis: progress and prospects. Dis Model Mech. 2017;10(9):1061-1074.

46. Morton JJ, Bird G, Refaeli Y, Jimeno A. Humanized Mouse Xenograft Models: Narrowing the Tumor-Microenvironment Gap. Cancer Res. 2016;76(21):6153-6158.

47. Choi Y, Lee S, Kim K, Kim SH, Chung YJ, Lee C. Studying cancer immunotherapy using patientderived xenografts (PDXs) in humanized mice. Exp Mol Med. 2018;50(8):99.

\section{Figure Captions}

\section{Figure 1:}

Patient Characteristics and summary of clinically relevant gene mutations from the patient nonsmall cell lung cancer (NSCLC) brain metastasis samples in which patient-derived xenografts (PDXs) were established. Age at diagnosis, gender, smoking pack-years, synchronous brain metastasis, yes or no, prior radiotherapy yes or no, programmed death-ligand 1 (PD-L1) immunohistochemistry (IHC) percent positive. 


\section{Figure 2:}

Histopathologic features of nine patient non-small cell lung cancer (NSCLC) brain metastases and corresponding NSCLC brain metastasis patient-derived xenografts (PDXs). Shown are photomicrographs hematoxylin and eosin (H\&E) of patient tumor, PDX passage 1 (P1) and passage 5 (P5) and thyroid transcription factor-1 (TTF-1) of patient tumor and passage 1 (P1). Images at $20 X$.

\section{Figure 3:}

Establishment of subcutaneous flank and orthotopic intracranial patient non-small cell lung cancer (NSCLC) brain metastasis xenografts. (A) Subcutaneous flank xenograft growth characteristics of University of Wisconsin (UW)-lung-2, UW-lung-5, UW-lung-16 and UW-lung18 and UW-lung-21. (B) Representative MR images and histology of UW-lung-2 and UW-lung16 orthotopic intracranial xenografts. (C) Pictures of established cell lines UW-lung-2 (passage 20) and UW-lung-16 (passage 20).

\section{Figure 4:}

RNAseq shows high correlation between patient and patient-derived xenografts (PDXs) tumor samples. (A) Correlation heatmap and hierarchical clustering of patient and PDX tumor samples. (B) Top 10 Gene Ontology (GO) terms as summarized by bar charts showing the $p$ value (lighter hue) and false discovery rate (FDR) q value (darker hue). Pathways are labeled along the $y$-axis; the number of genes annotated within each pathway is indicated in parentheses. (C-E) Principal component analysis (PCA) clustering of patient and PDX tumor 
RNA samples. (F) Top GO terms overrepresented in PC2; showing the number of genes (count), the q-value and the percent hits. (G) Pearson correlation coefficient of all genes on the $\mathrm{x}$-axis and after removing the top $1 \%$ (219 genes) contributing to PC2.

\section{Figure 5:}

Conservation of mutational landscape. (A) Venn diagram of all variants detected in UW-lung-2 patient brain metastasis, brain PDX and flank PDX. (B) Scatterplots displaying the correlation between patient brain metastasis variant allele frequency (VAF) and flank PDX, patient brain metastasis VAF and brain PDX and flank PDX and brain PDX. The $\mathrm{R}^{2}$ value is represented by the black value in the lower right-hand corner of each plot.

\section{Figure 6:}

Therapeutic effects of radiation, selumitinib and savolitinib on xenograft growth delay. (A) Radiation-induced tumor growth delay curves of UW-lung-2, UW-lung-5, UW-lung-16, UW-lung18 and UW-lung-21. Radiation was given as 2 Gy per fraction for either 5 days or 10 days as indicated. (B) KRAS G12C mutated UW-lung -2 and UW-lung-16 subcutaneous flank xenografts treated with vehicle or selumitinib $25 \mathrm{mg} / \mathrm{kg}$ BID for 10 days. (C) Immunohistochemistry (IHC) of UW-lung-2 and UW-lung-16 tumor sections showing modulation of KRAS-downstream signaling ( $p$-Erk and p-S6) following a one-time dose of $25 \mathrm{mg} / \mathrm{kg}$ BID of selumitnib, collected at 24 hours. (D) MET exon 14 mutated UW-lung-21 subcutaneous flank xenografts treated with vehicle, savolitinib $2.5 \mathrm{mg} / \mathrm{kg}$, radiation or radiation plus savolitinib $2.5 \mathrm{mg} / \mathrm{kg}$ for 10 days. (E) Immunohistochemistry of UW-lung-21 tumor sections showing modulation of MET signaling (MET, p-MET, p-Erk and p-S6) and decrease in Ki-67 following a single dose of $2.5 \mathrm{mg} / \mathrm{kg}$ of savolitinib, collected at 24 hours. Points, mean tumor volume in mice after treatment; bars, SEM. The scale bar represents $50 \mathrm{~mm}$ and all images are to the same scale. 
bioRxiv preprint doi: https://doi.org/10.1101/2020.06.02.130062; this version posted October 1,2020. The copyright holder for this preprint

(which was not certified by peer review) is the author/funder, who has granted bioRxiv a license to display the preprint in perpetuity. It is made available under aCC-BY-NC-ND 4.0 International license.

\section{Supplemental Tables}

Table S1: Detailed mutation list

Table S2: Short tandem repeat analysis loci

Table S3: KEGG (Kyoto Encyclopedia of Genes and Genomes) Pathways

Table S4: Gene Ontology Terms 


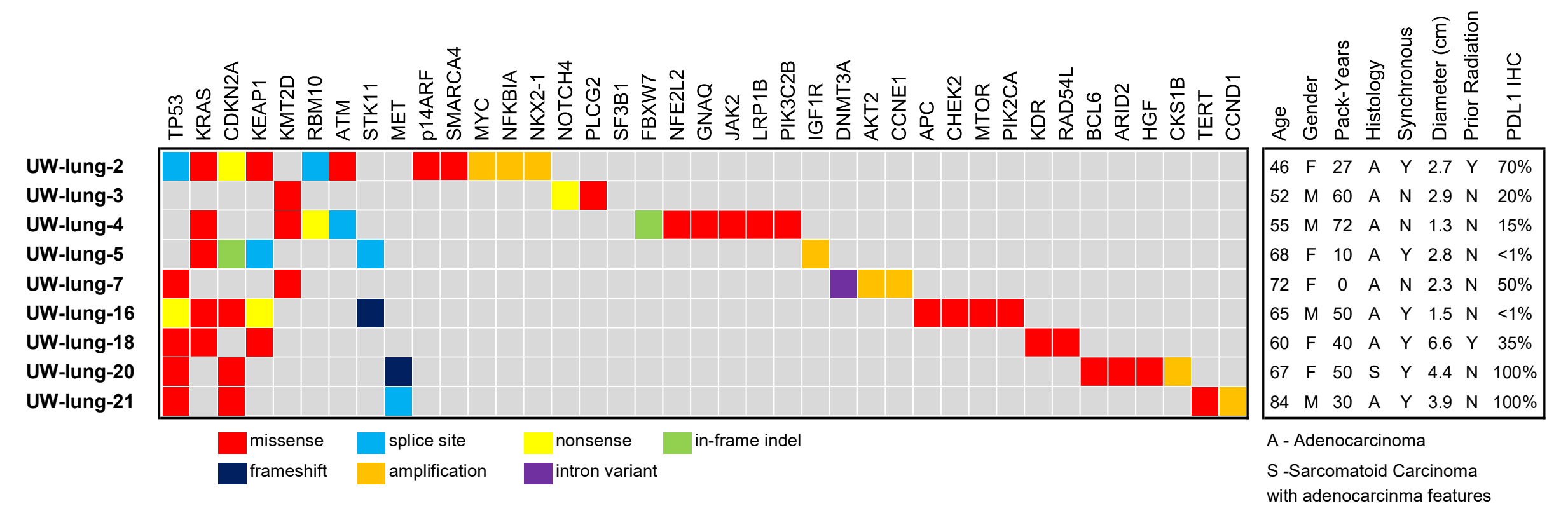


A

UW-lung-2

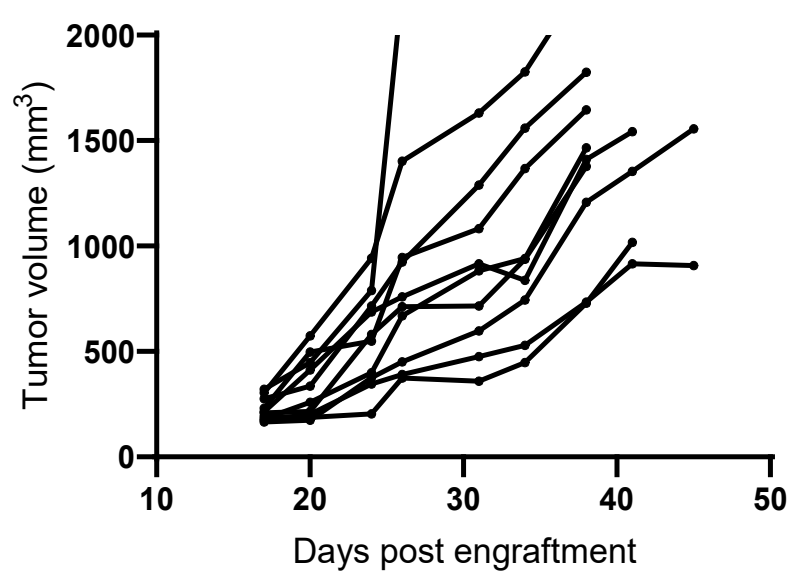

UW-lung-18

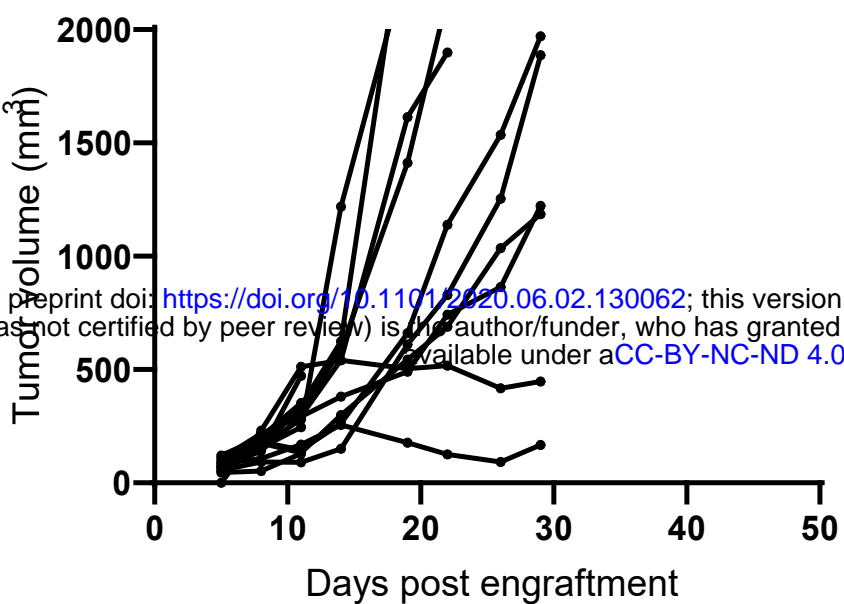

UW-lung-5

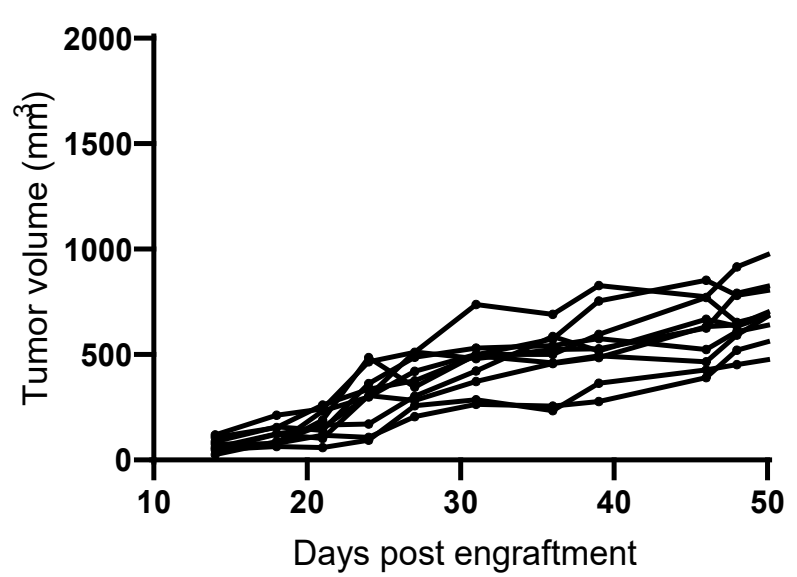

UW-lung-21

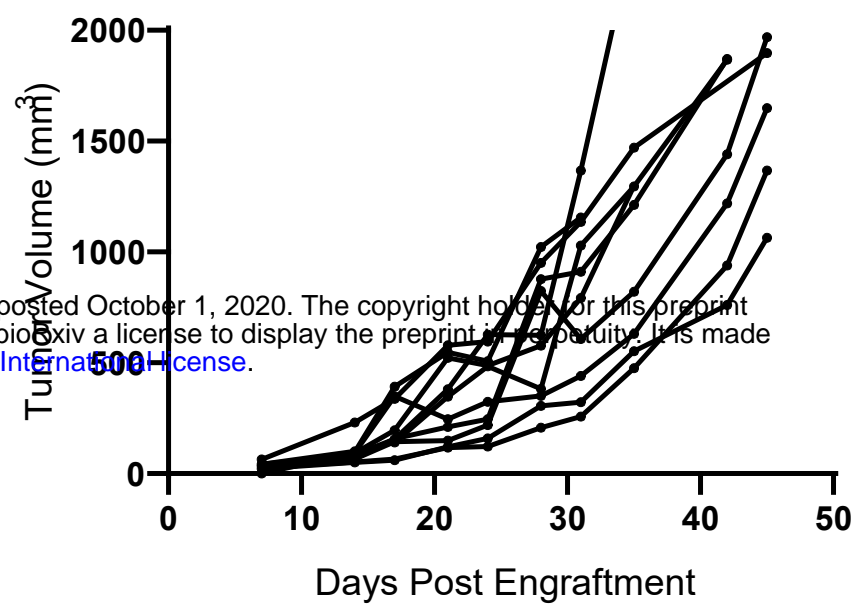

UW-lung-16

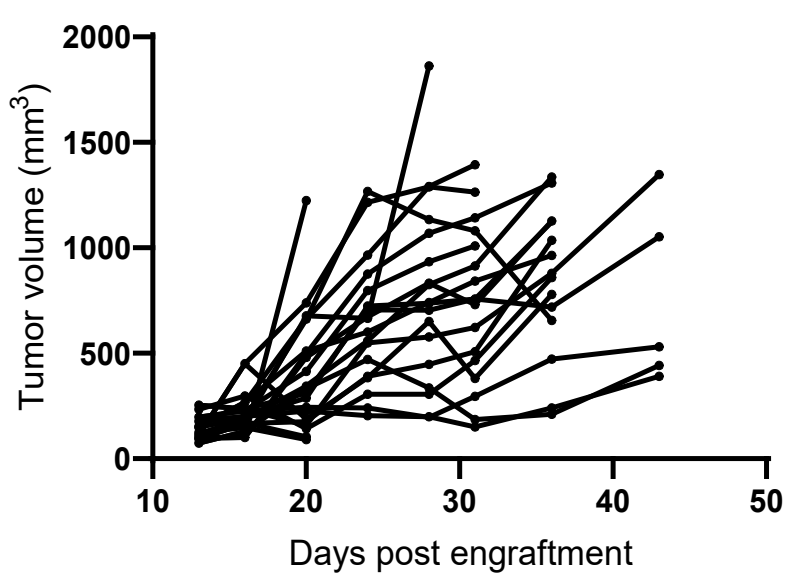

B

\section{UW-lung-2}
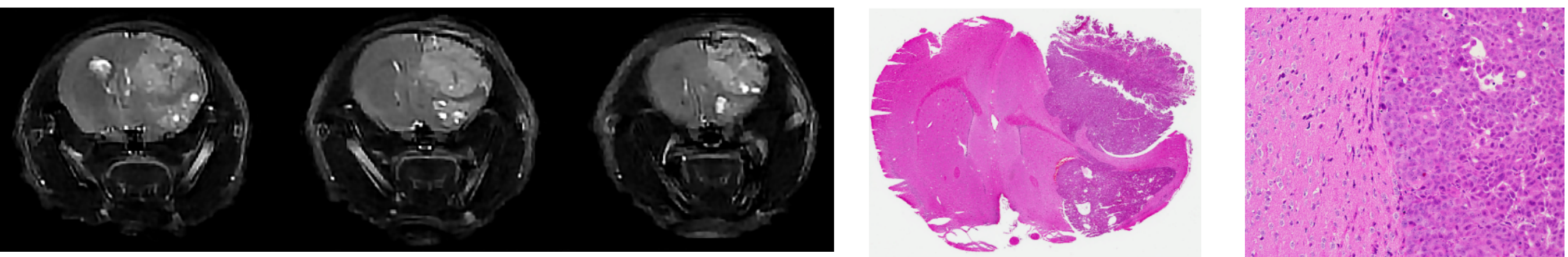

UW-lung-16
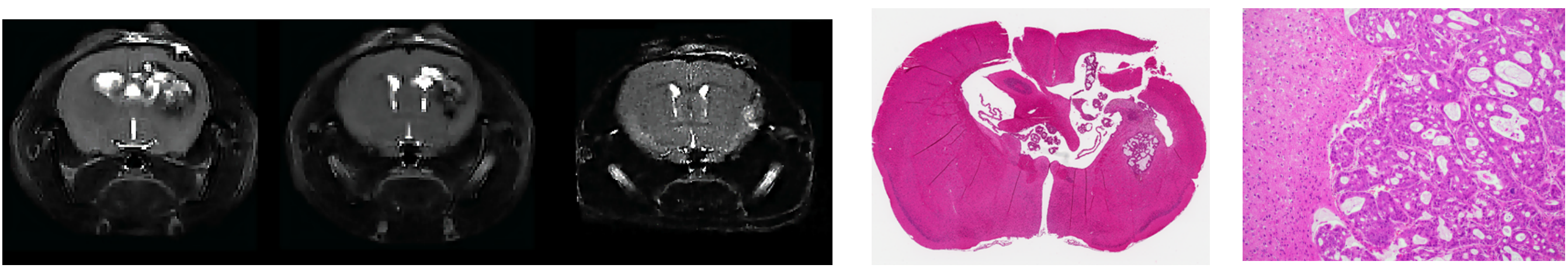

C

UW-lung-2

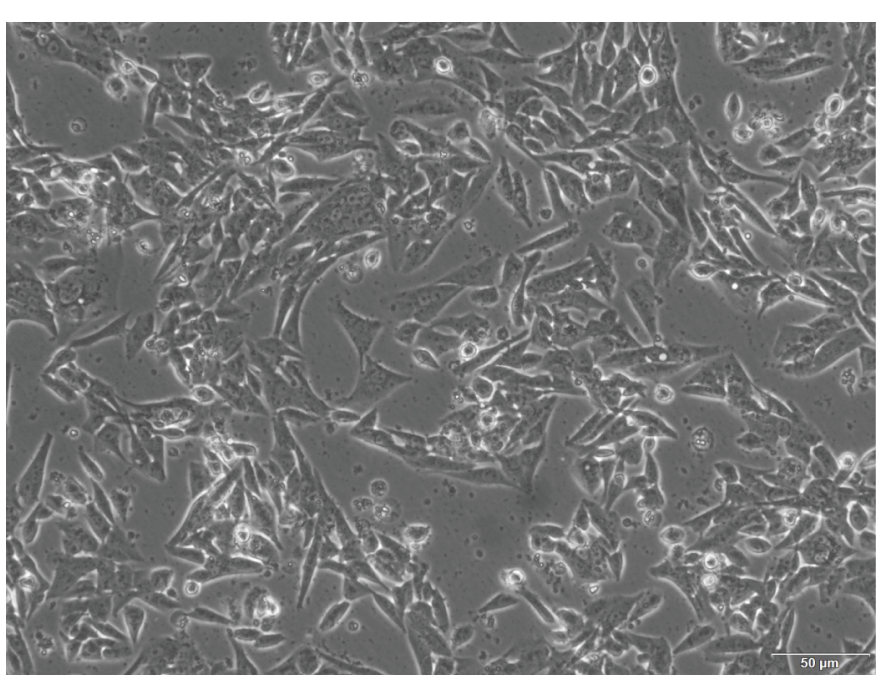

UW-lung-16

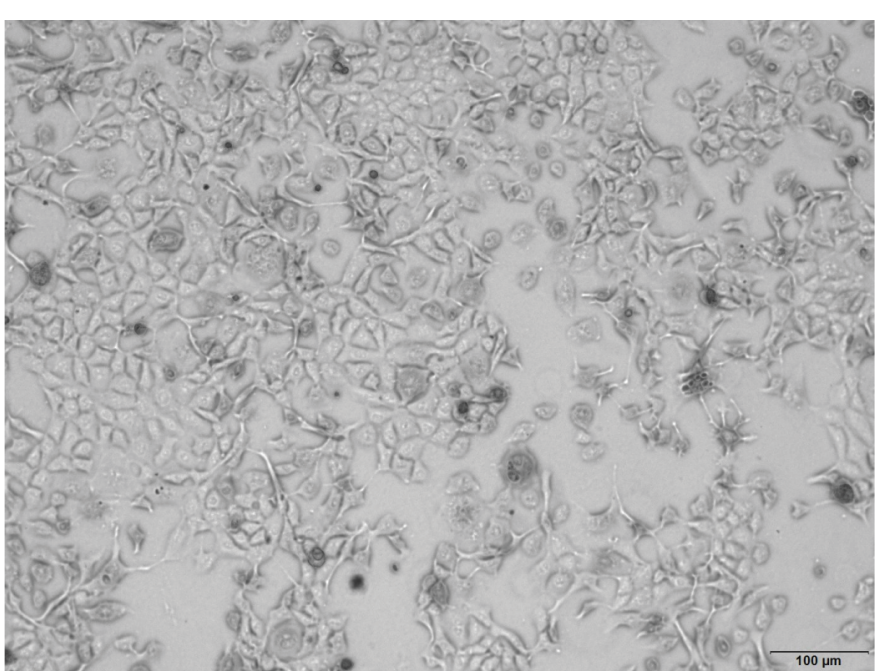




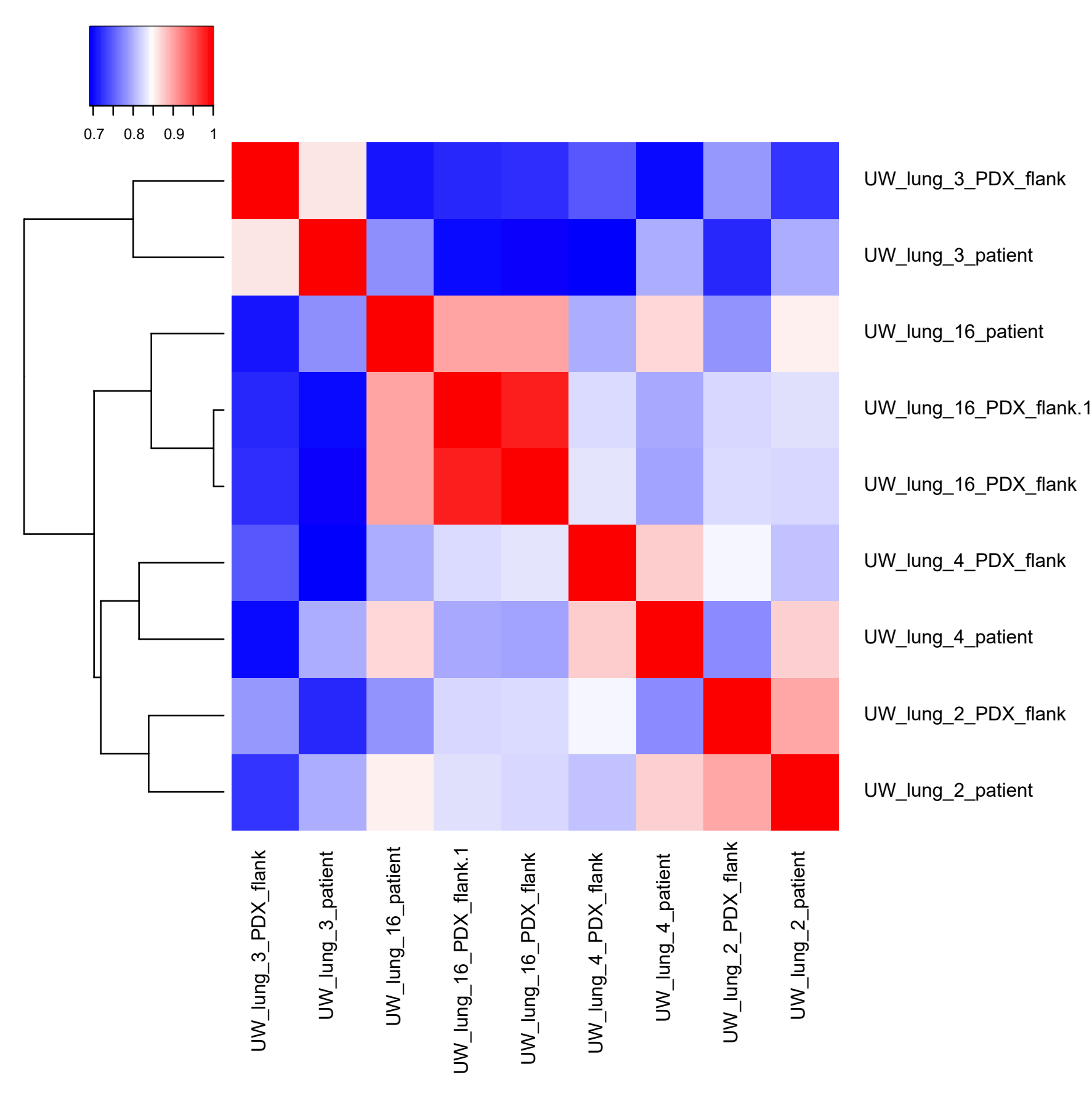

C
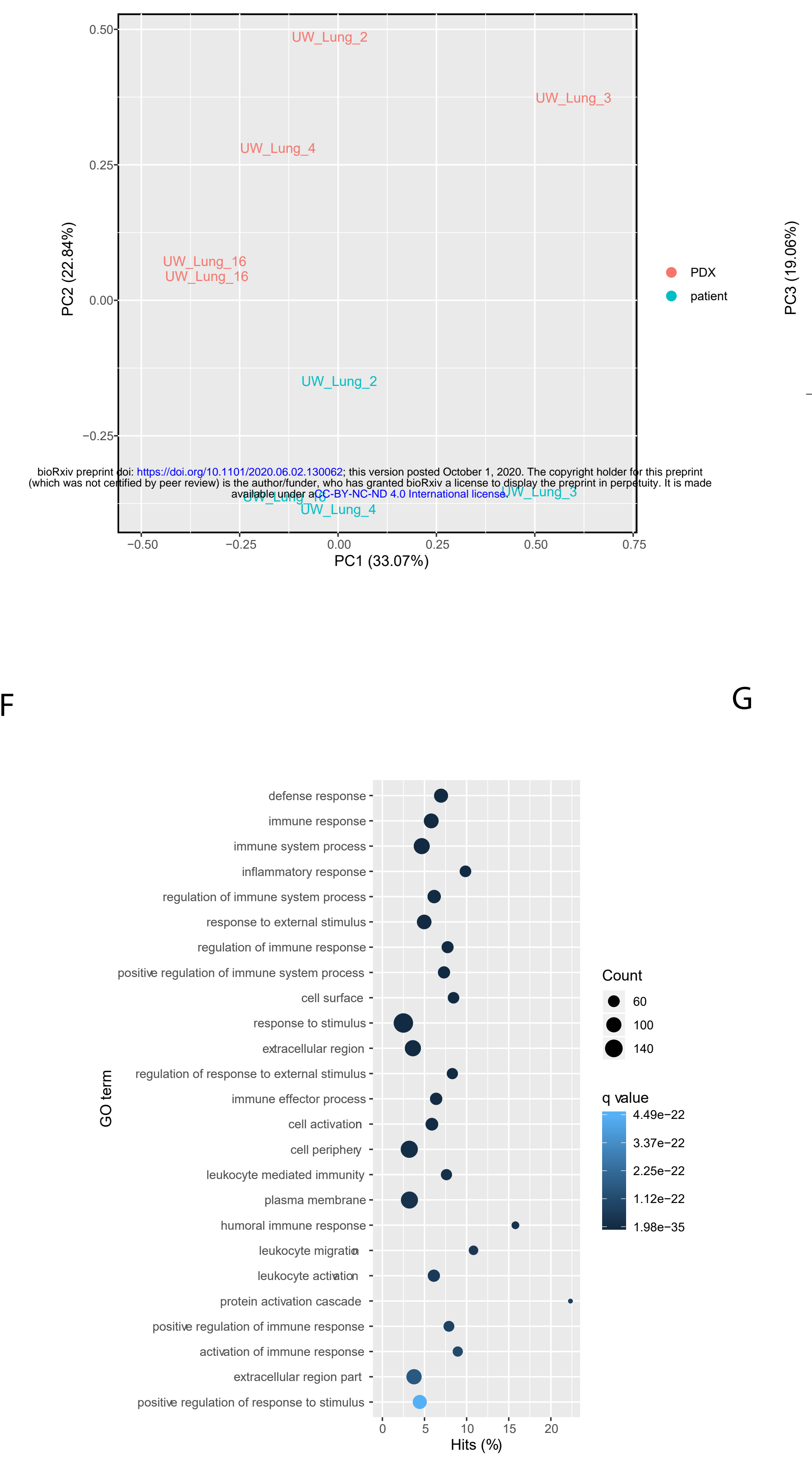
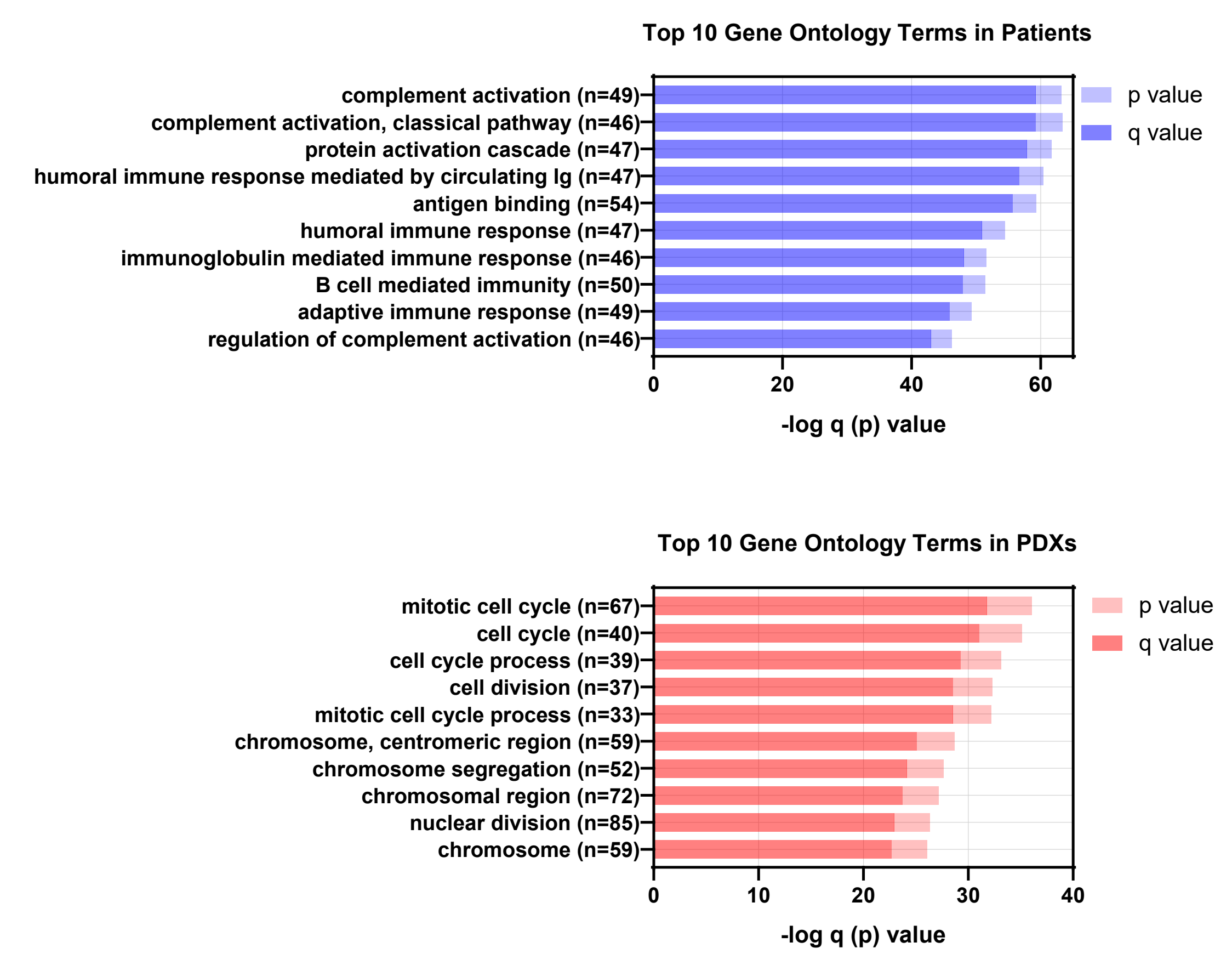

D

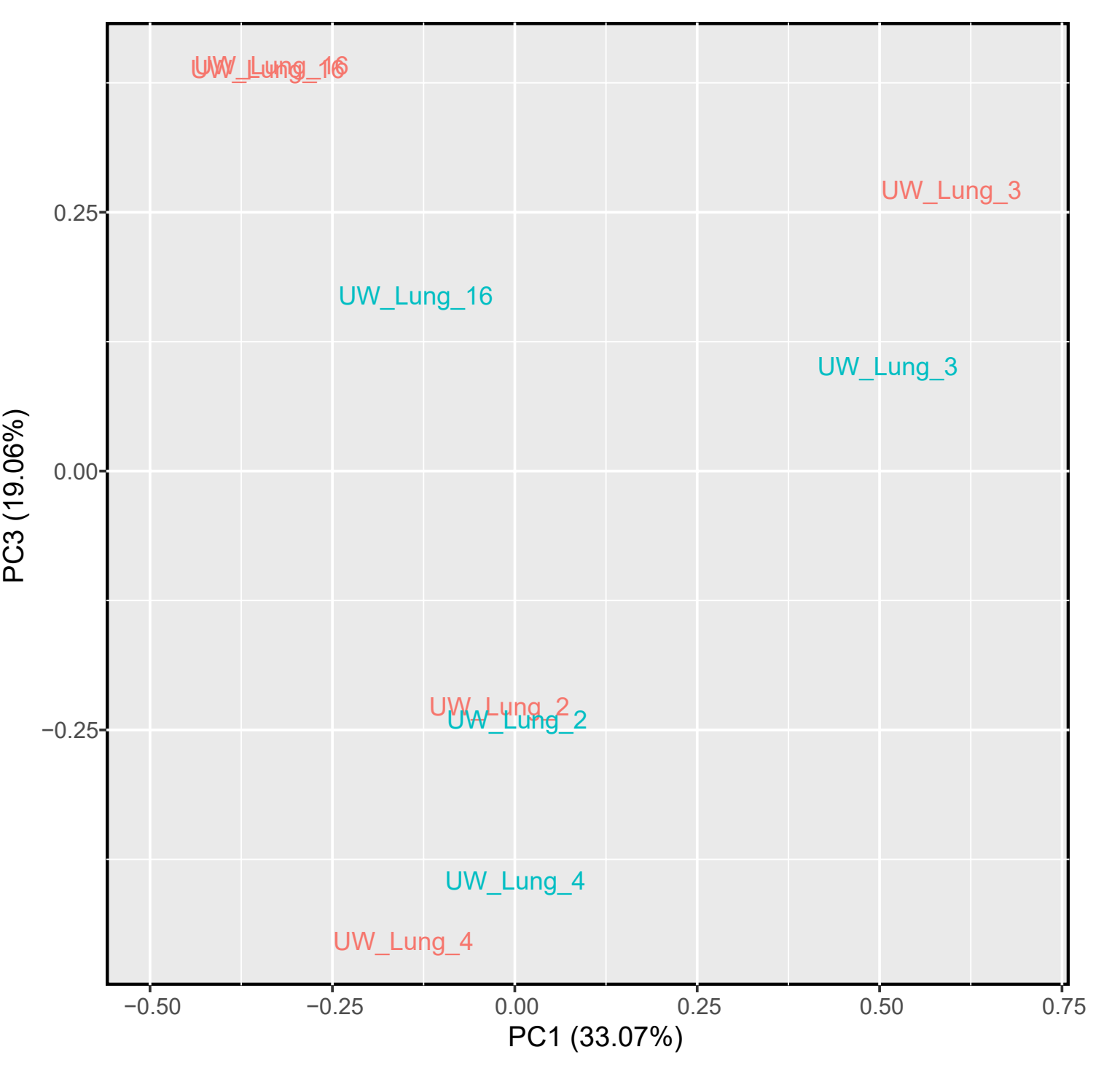

G

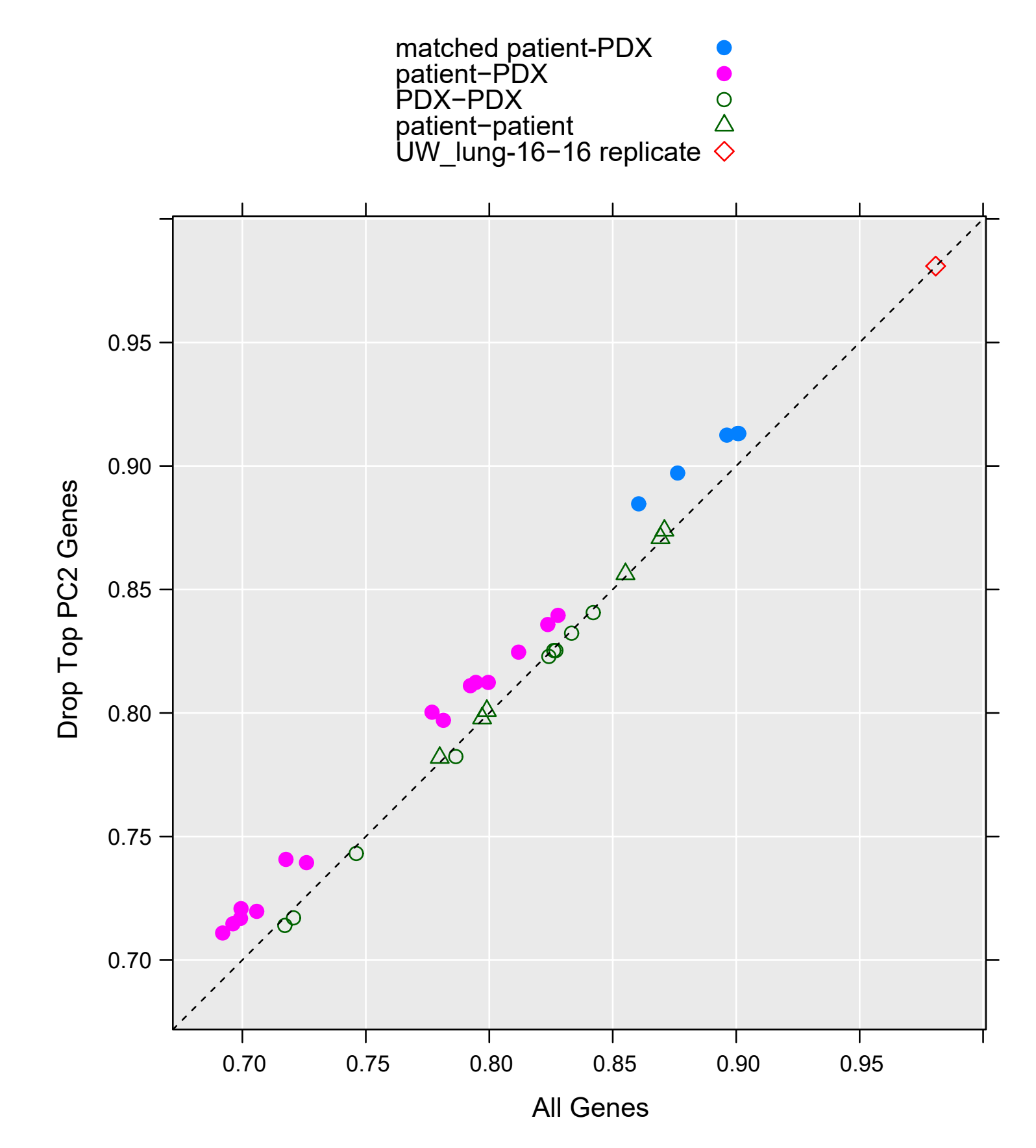

G
E

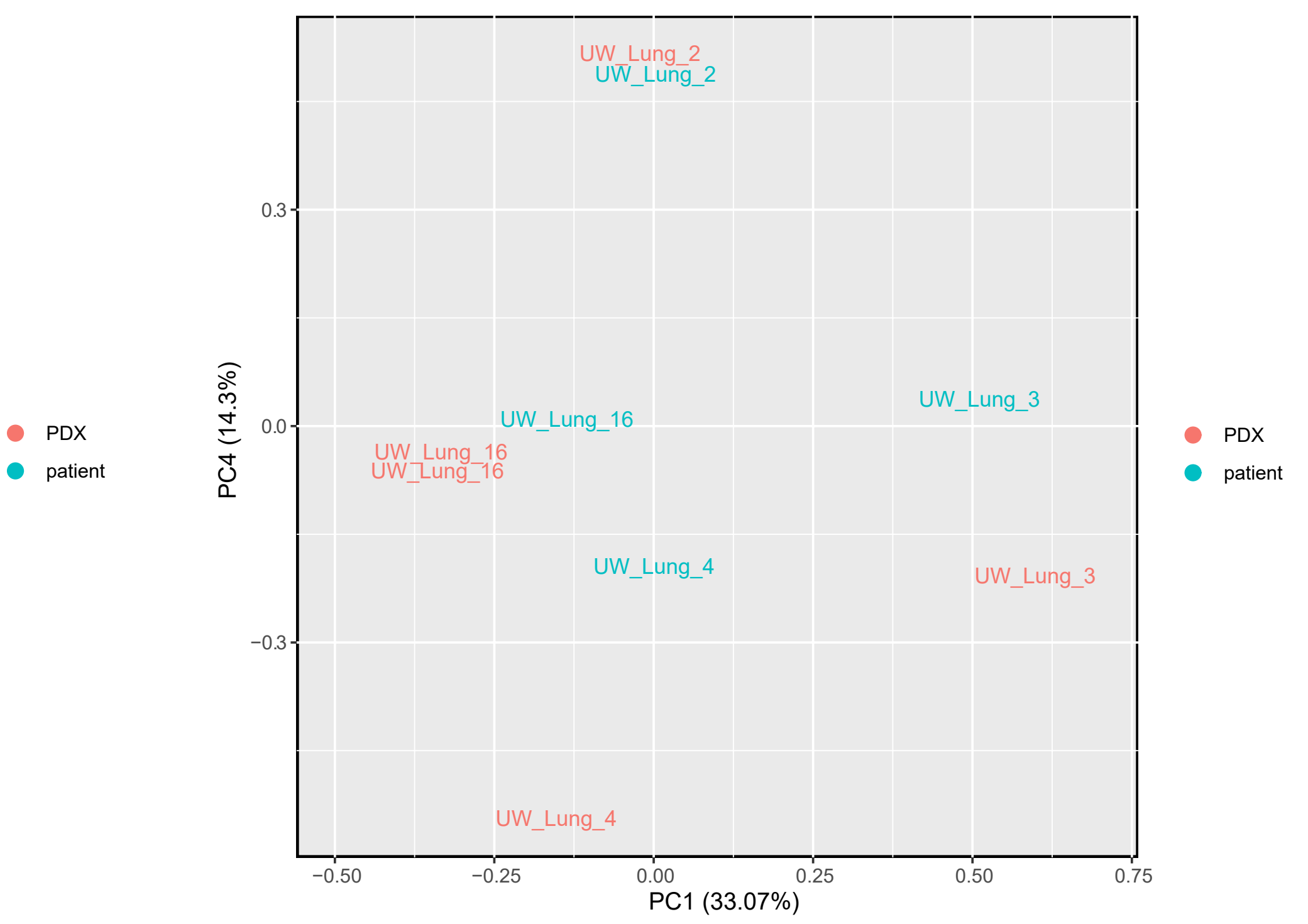


A UW-lung-2 Patient UW-lung-2 PDX Flank

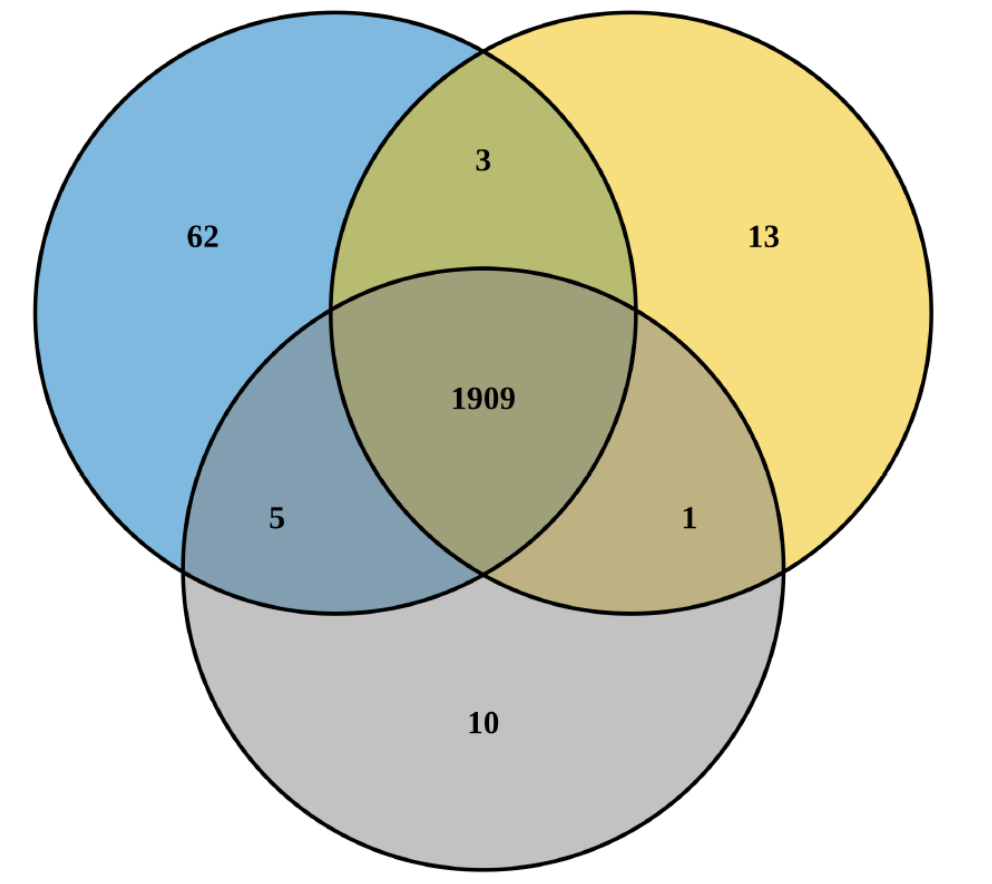

UW-lung-2 PDX Brain
UW-lung-2

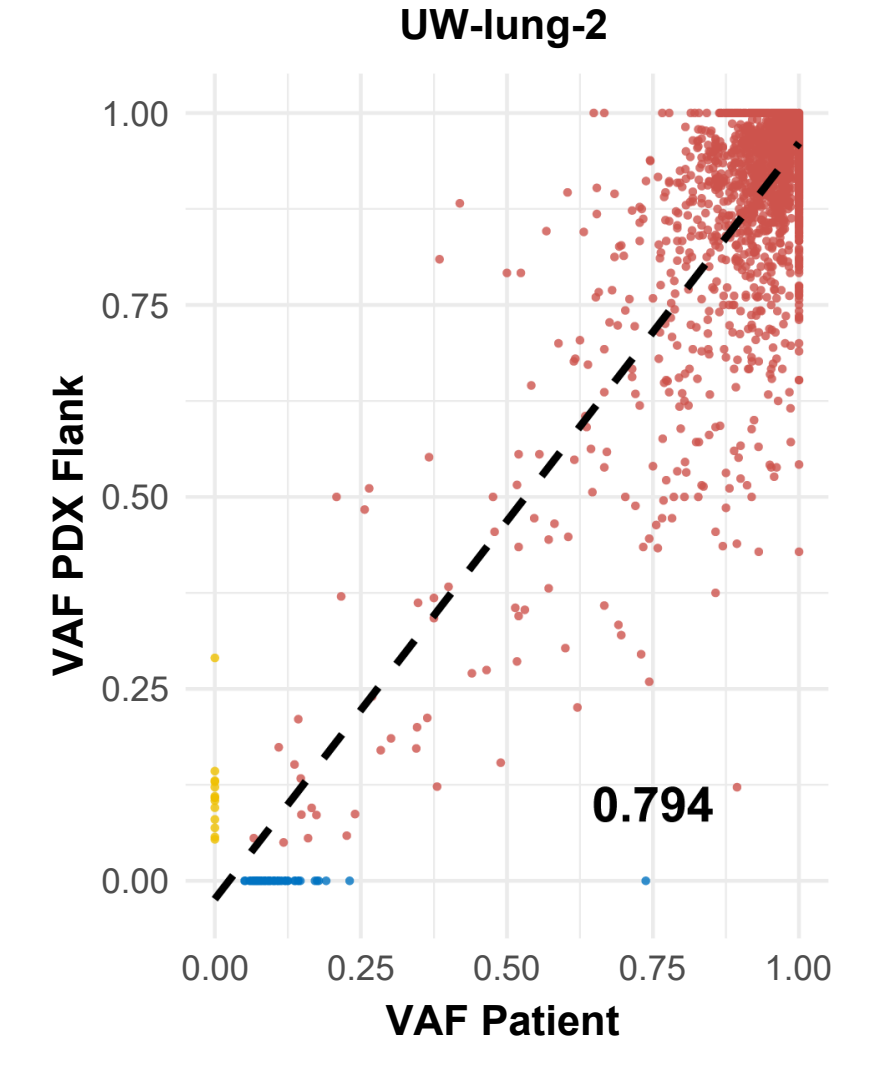

UW-lung-3

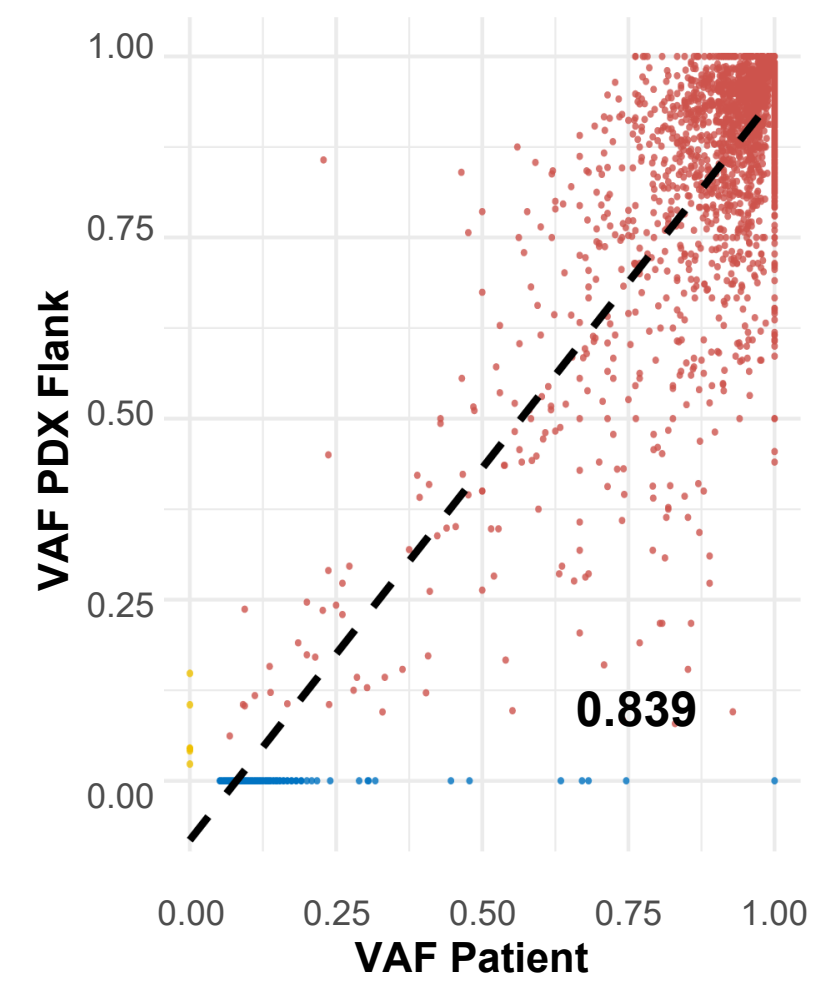

UW-lung-2

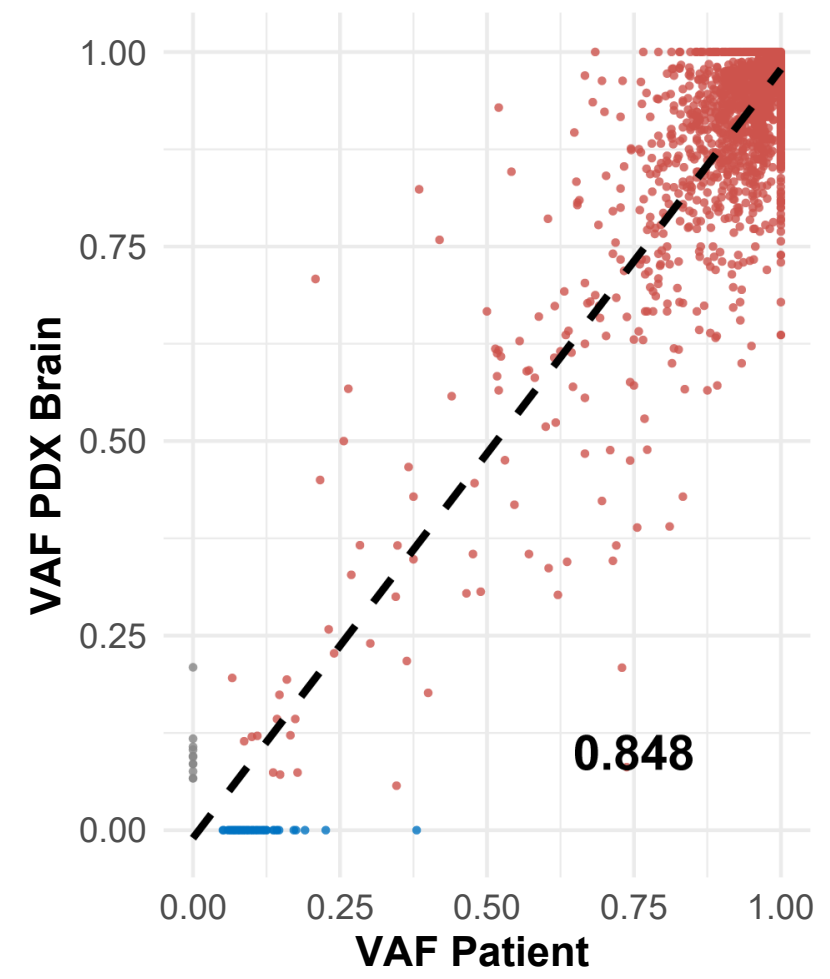

Common

- Patient

- Patient

PDXBrain

UW-lung-4

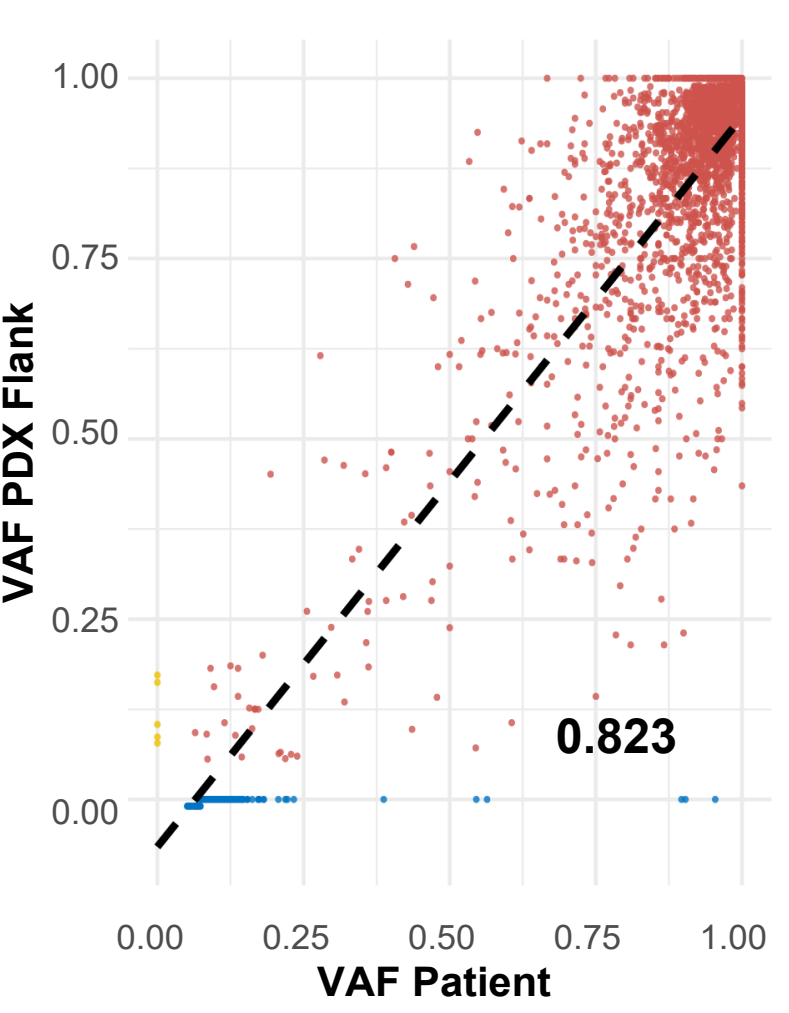

- Common

- PDX Flank

- Patient

- Common

- PDX Flank

- Patient

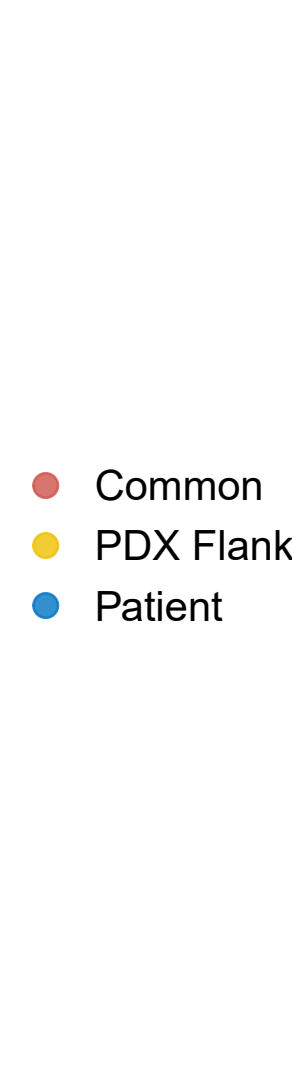

UW-lung-2

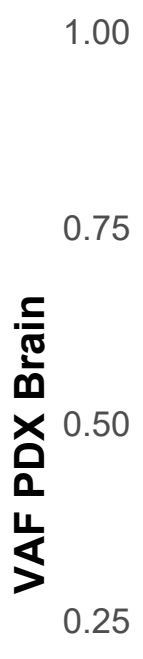

PDX Flank
- Common

- PDX Flank

- Patient
0.819

$0.00 \quad 0.25 \quad 0.50 \quad 0.75$ 

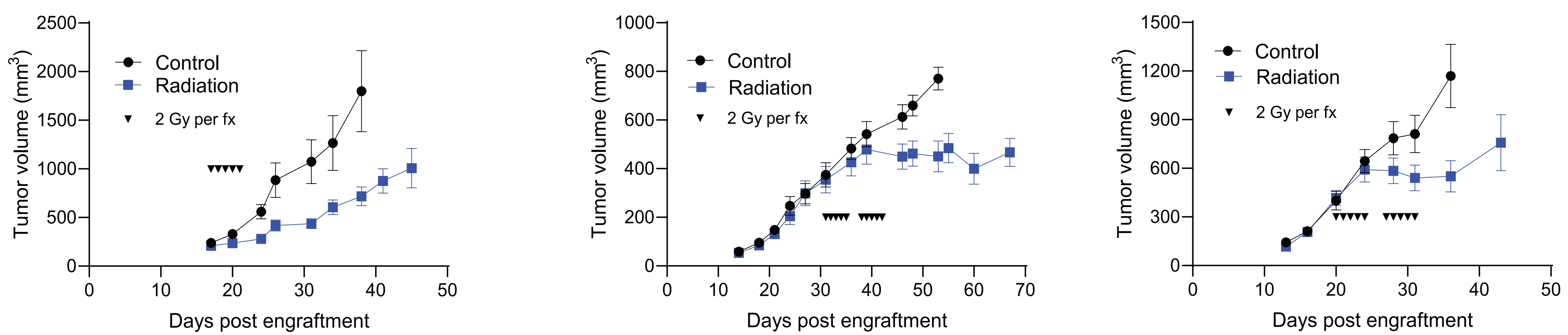

UW-lung-18

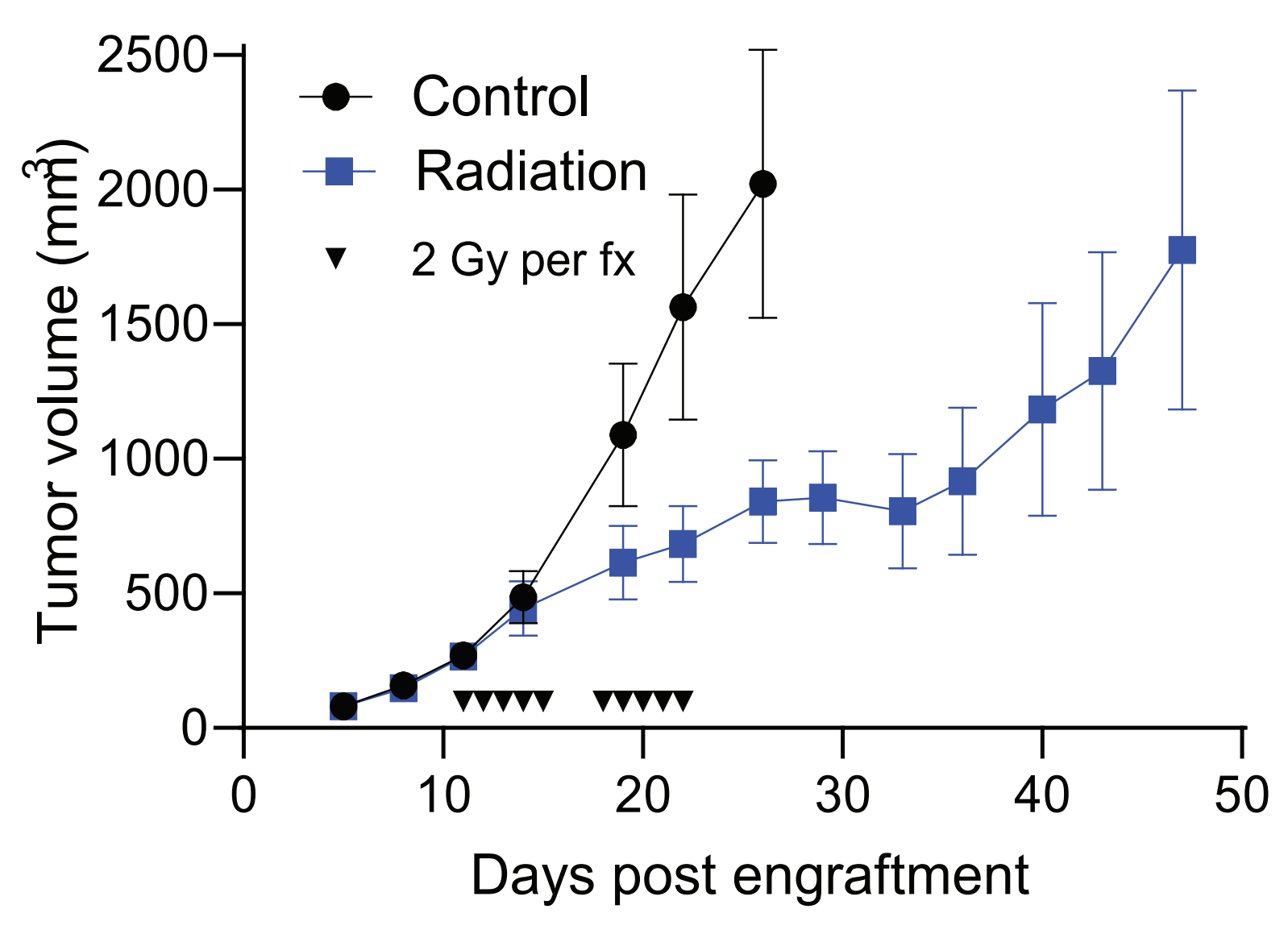

UW-lung-21

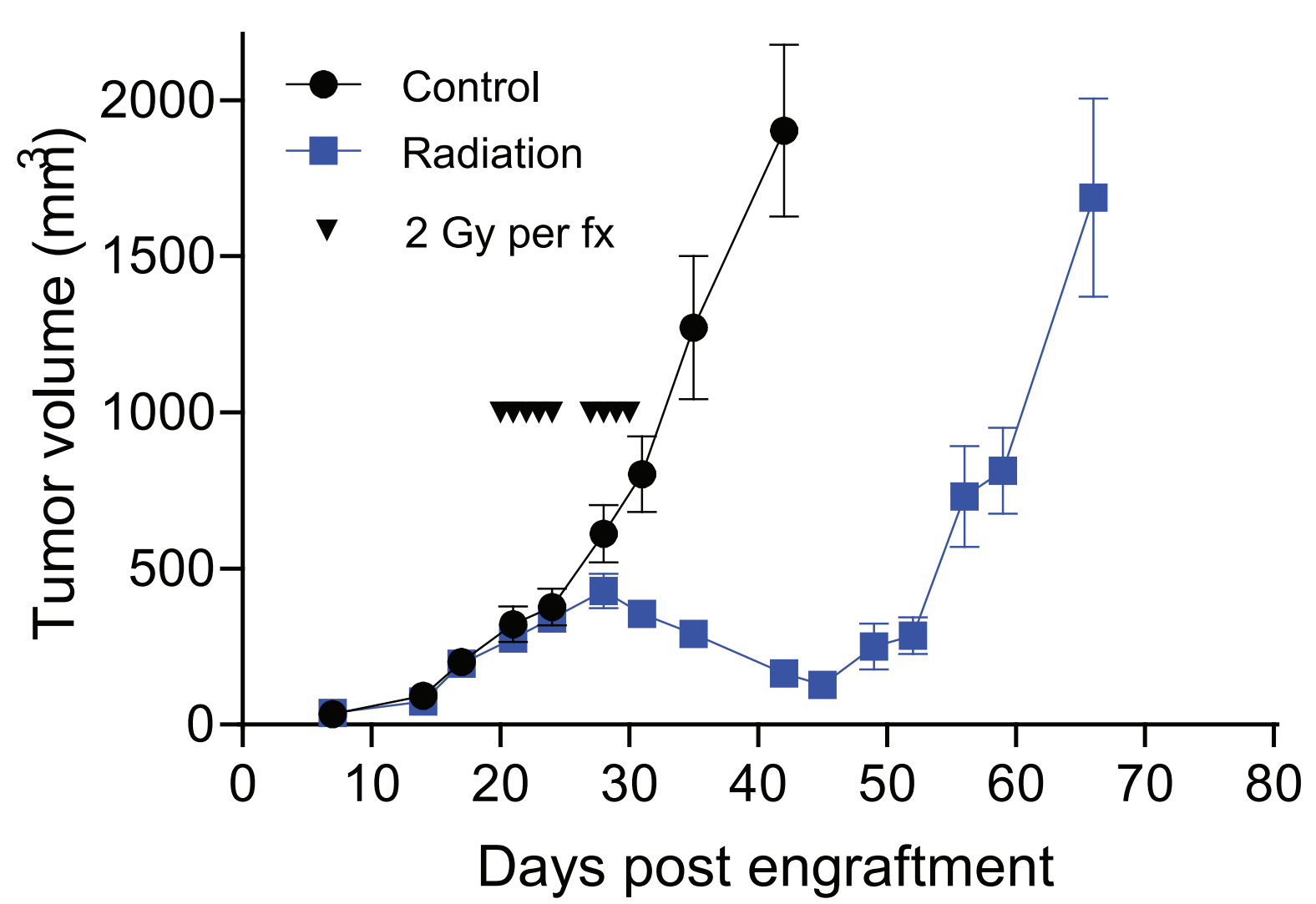

UW-lung-2

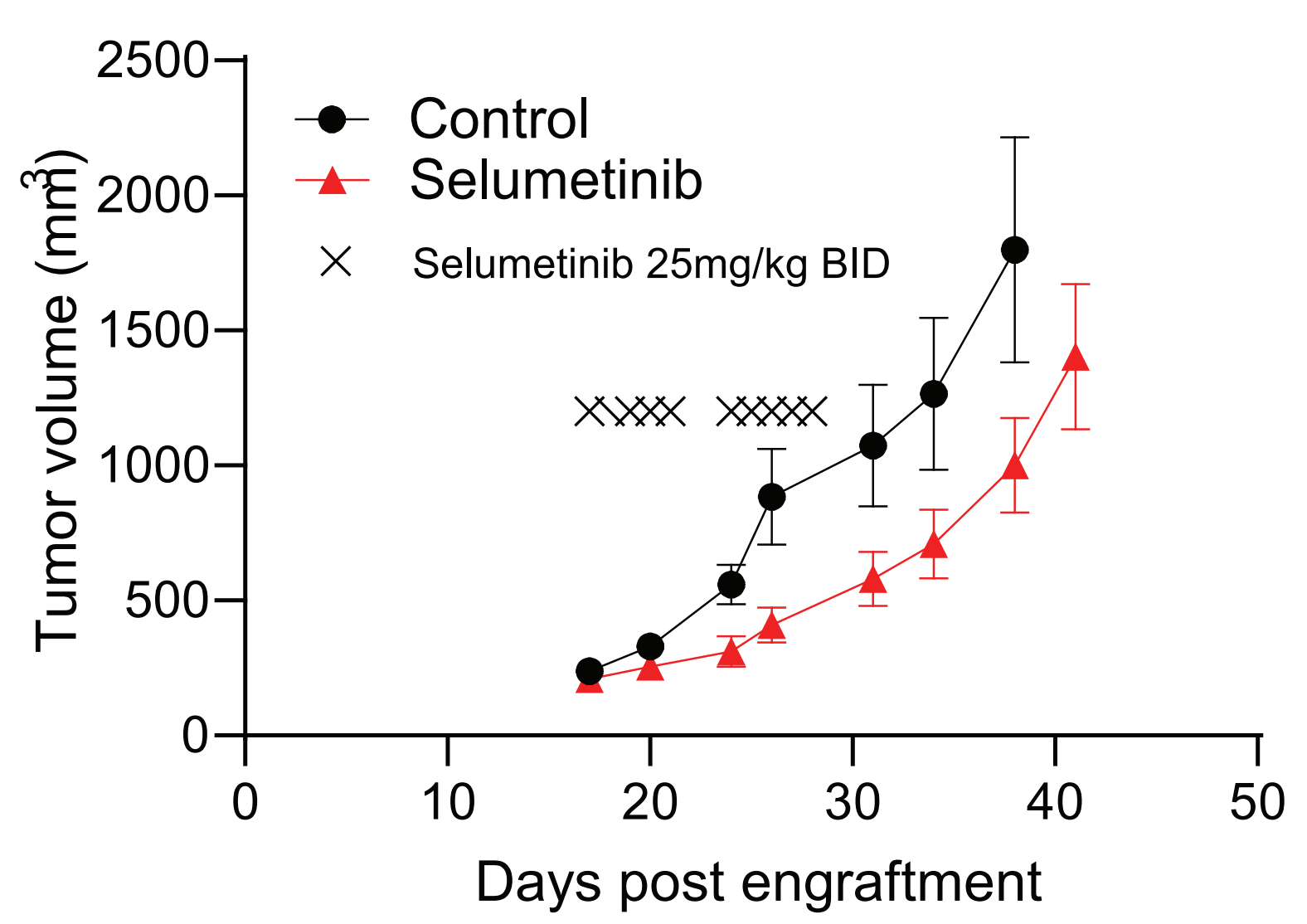

C

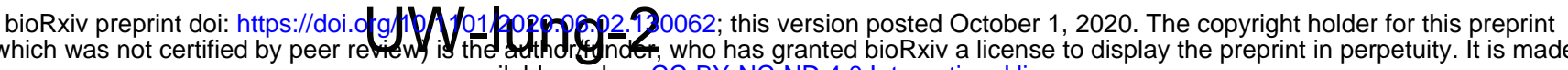

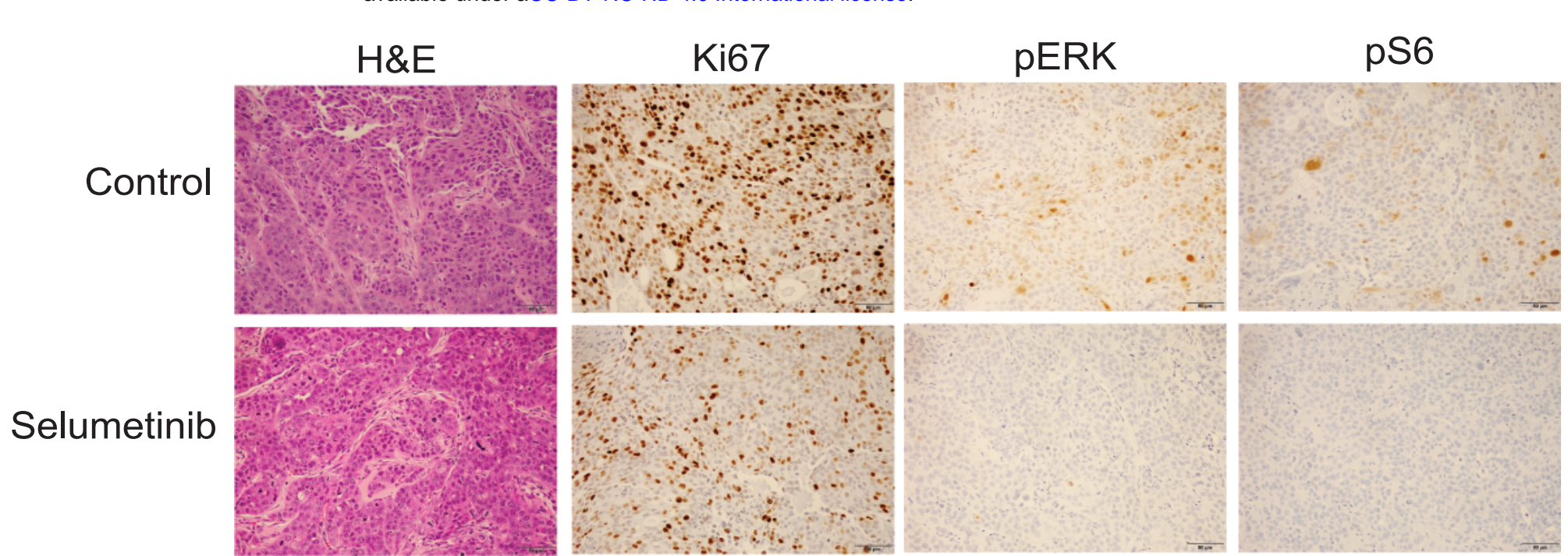

E

UW-lung-21

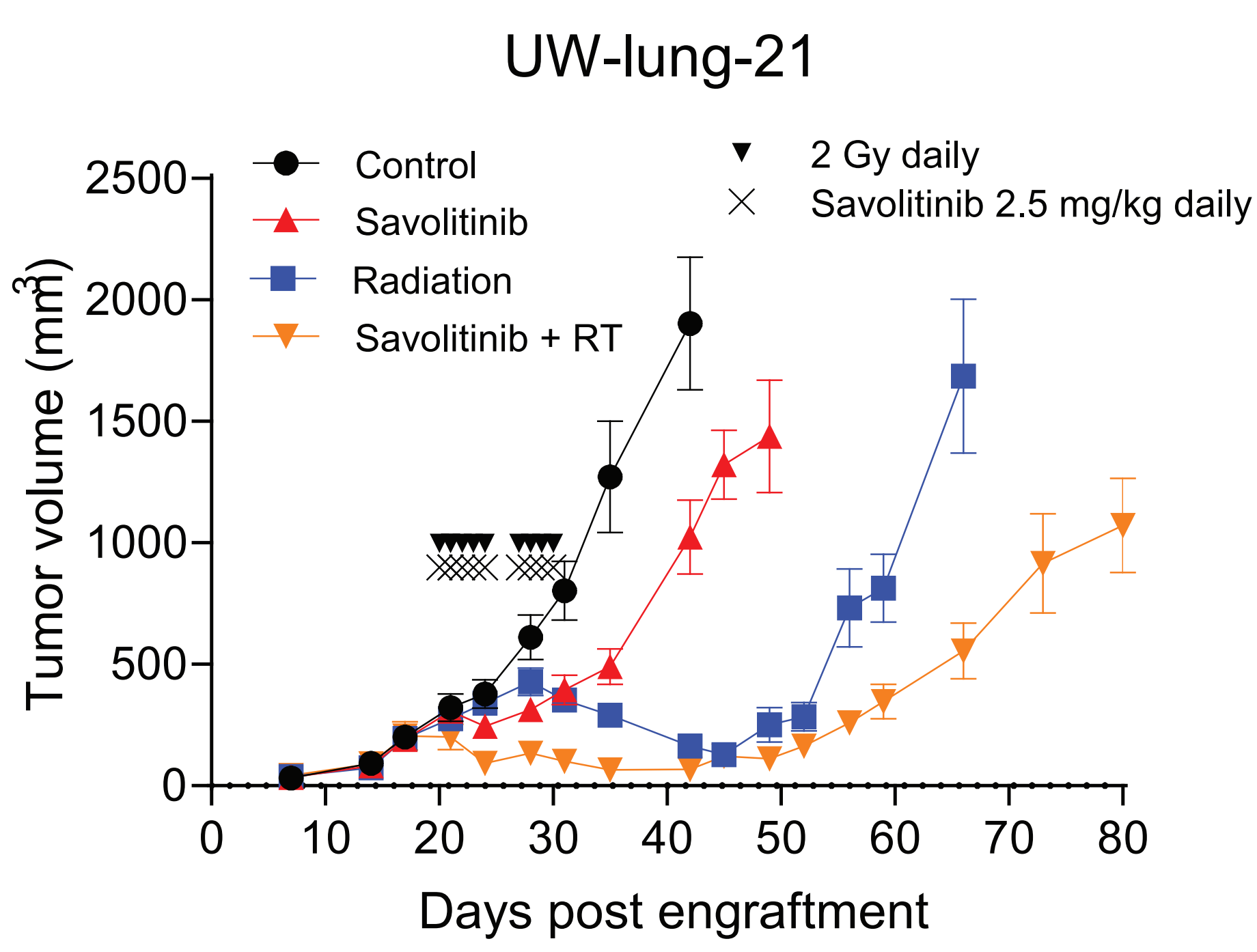

UW-lung-16

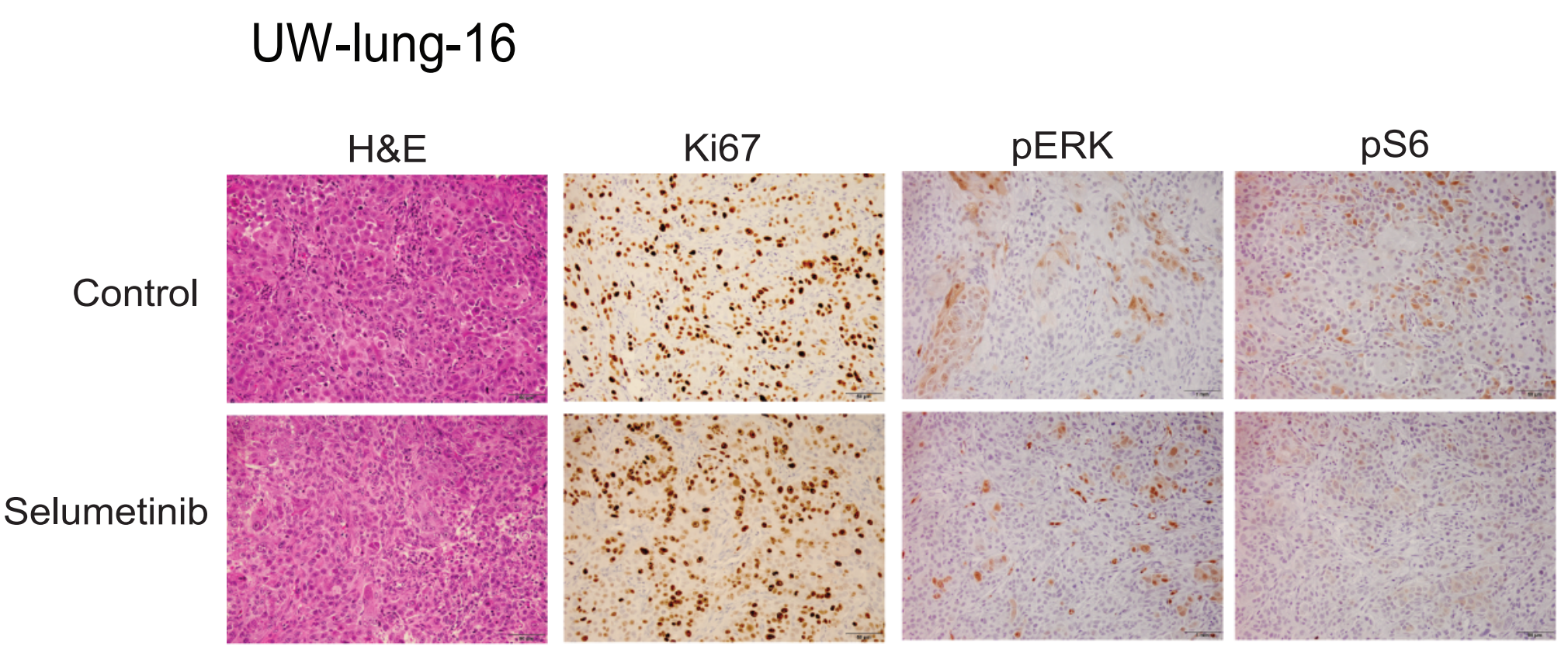

UW-lung-16

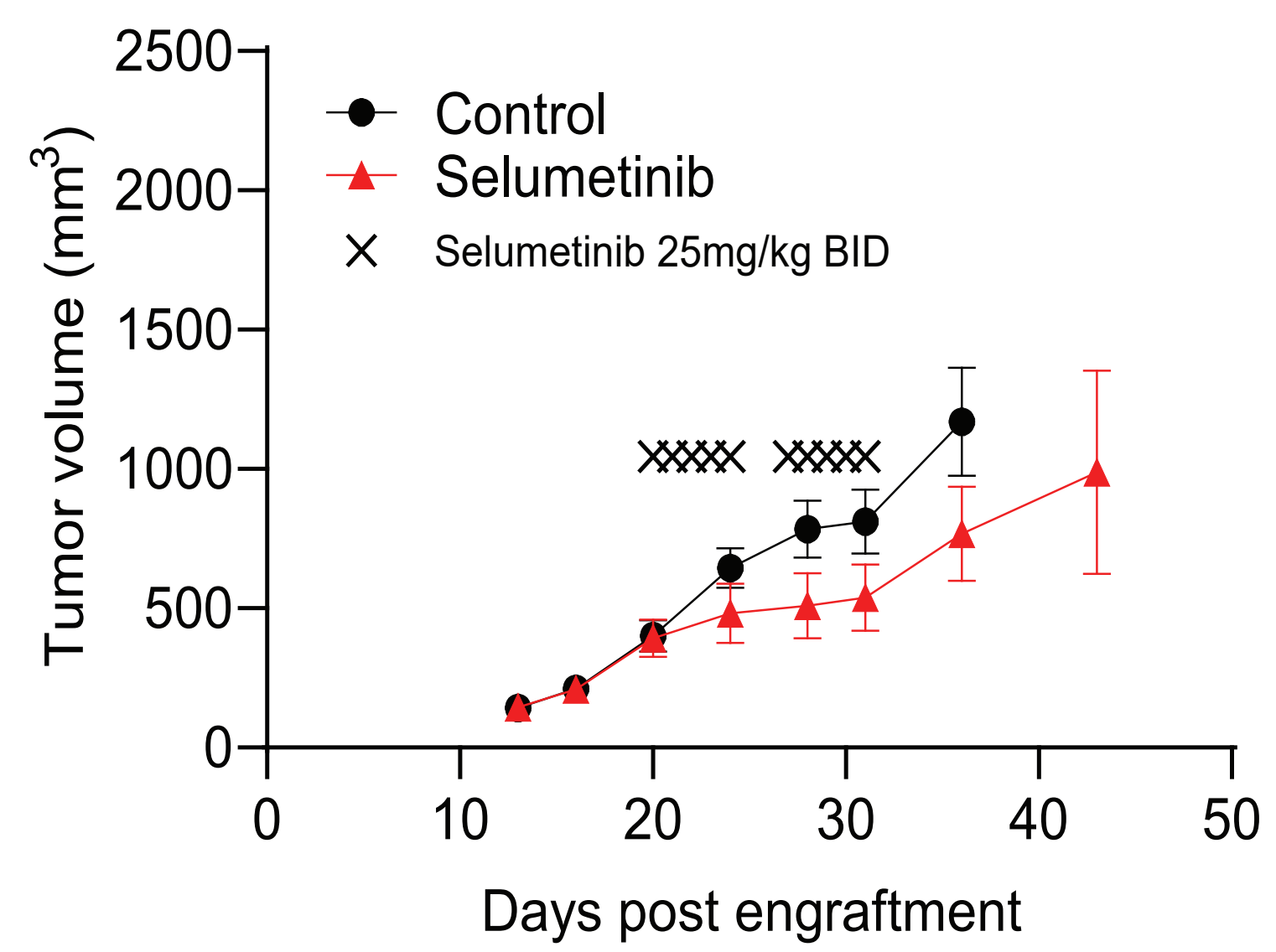

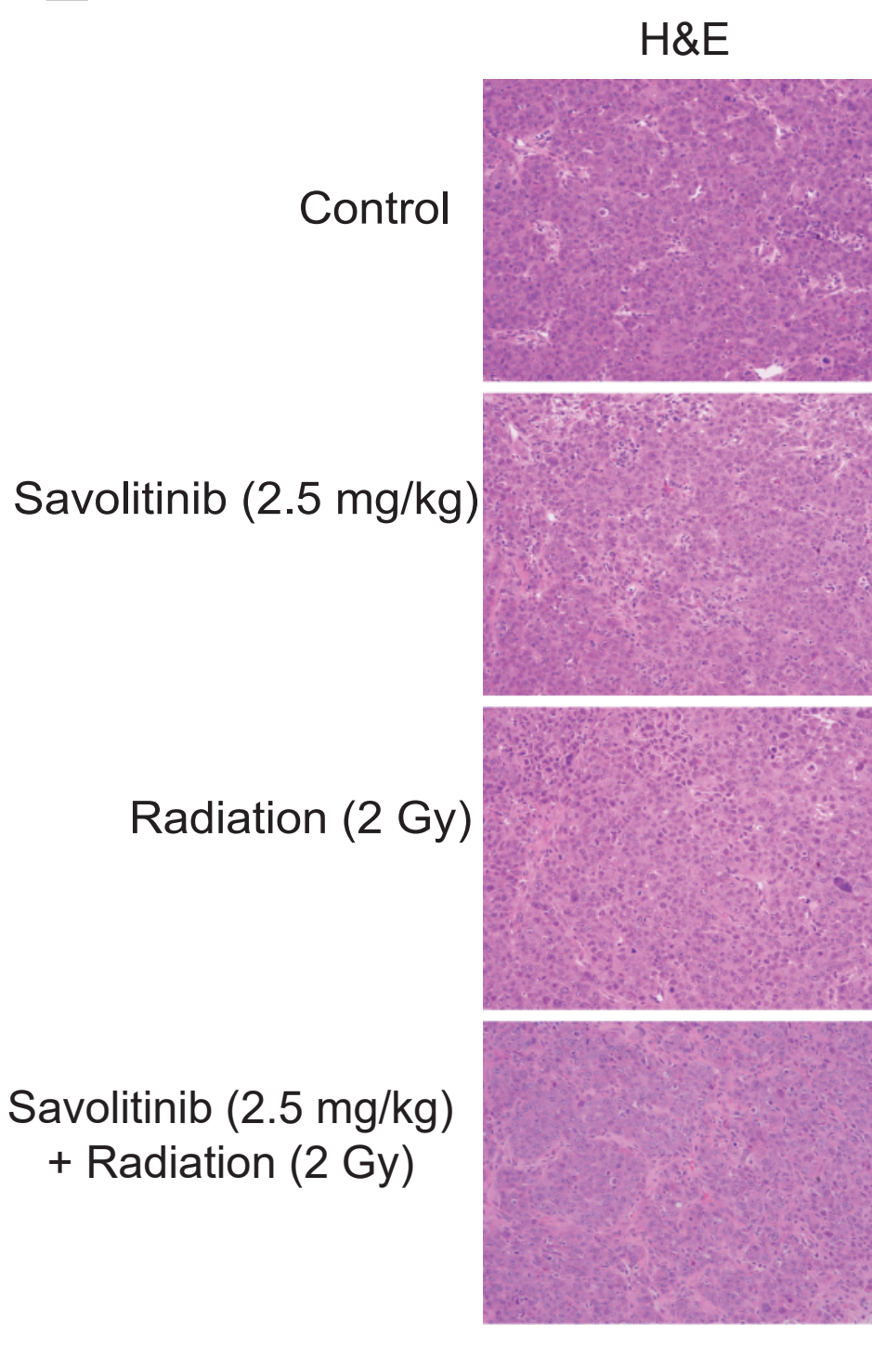

MET

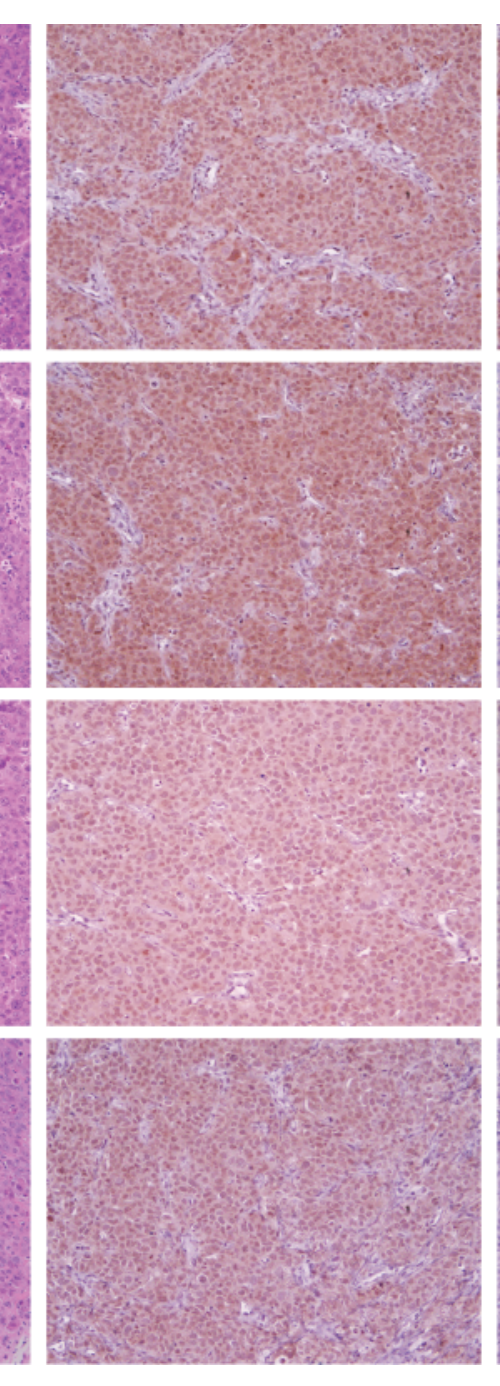

PMET

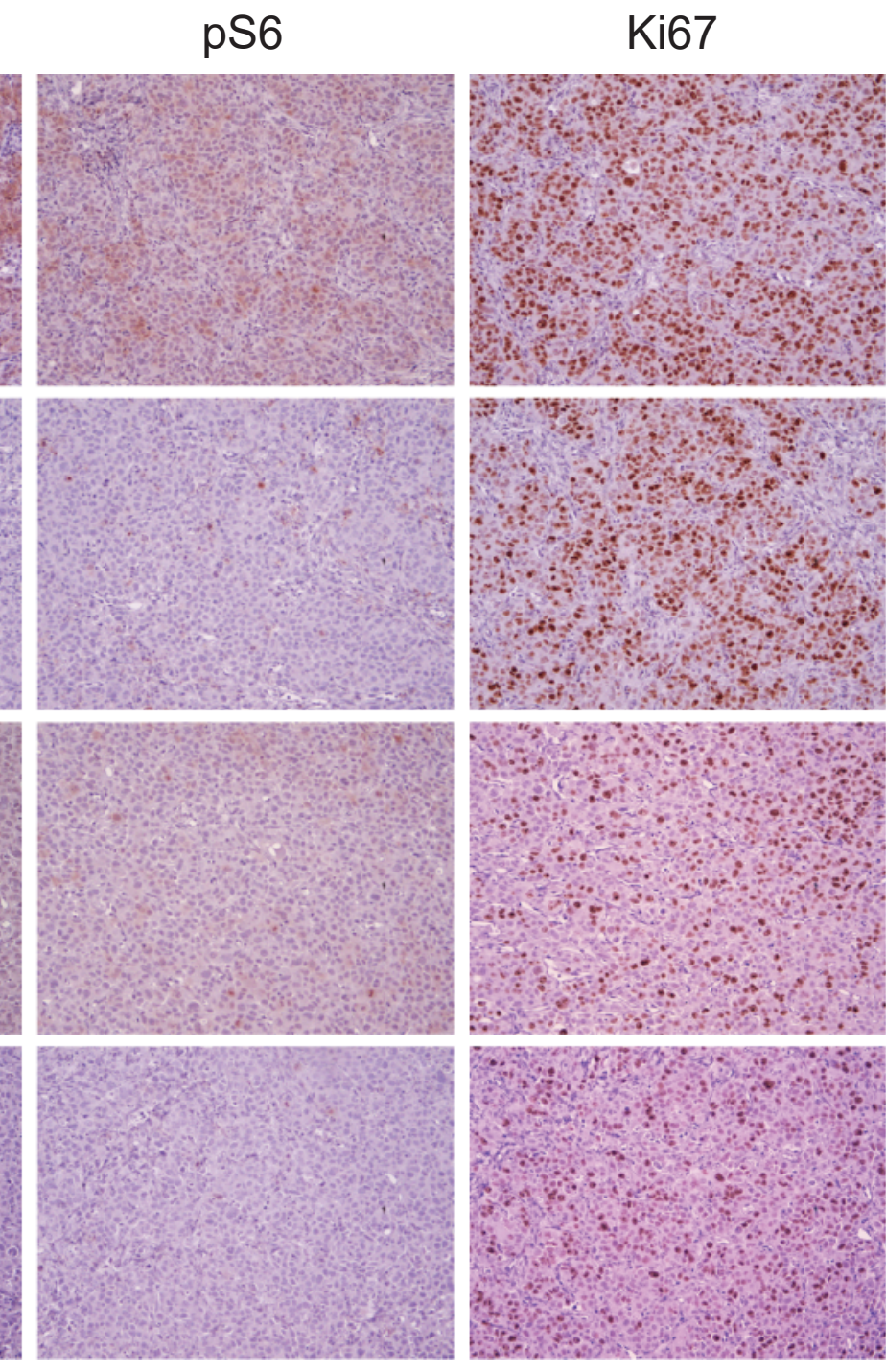

